Submitted to AJ

\title{
The Vast Population of Wolf-Rayet and Red Supergiant Stars in M101: I. Motivation and First Results
}

\author{
Michael M. Shara ${ }^{1}$, Joanne L. Bibby ${ }^{1,2}$, David Zurek ${ }^{1}$, Paul A. Crowther ${ }^{3}$, Anthony F.J. \\ Moffat $^{4}$, and Laurent Drissen ${ }^{5}$
}

\begin{abstract}
M101 is an ideal target in which to test predictions of massive star birth and evolution. The large abundance gradient across M101 (a factor of 20) suggests that many more WR stars must be found in the inner parts of this galaxy than in the outer regions. Many H II regions and massive star-forming complexes have been identified in M101; they should be rich in WR stars, and surrounded by RSG stars. Finally, the Wolf-Rayet stars in M101 may be abundant enough for one to explode as a Type Ib or Ic supernova and/or GRB within a generation. The clear identification of the progenitor of a Type Ib or Ic supernova as a WR star would be a major confirmation of current stellar evolution theory.

Motivated by these considerations, we have used the Hubble Space Telescope to carry out a deep, He II optical narrowband imaging survey of the massive star populations in the ScI spiral galaxy M101. Combined with archival broadband images, we were able to image almost the entire galaxy with the unprecedented depth and resolution that only HST affords.

We describe the extent of the survey and our images, as well as our data reduction procedures. A detailed study of a field east of the center of M101, containing the giant star-forming region NGC 5462, demonstrates how we find
\end{abstract}

\footnotetext{
${ }^{1}$ Department of Astrophysics, American Museum of Natural History, Central Park West and 79th Street, New York, NY 10024-5192

${ }^{2}$ Jeremiah Horrocks Institute for Maths, Physics \& Astronomy, University of Central Lancashire, Preston, PR1 2HE, United Kingdom

${ }^{3}$ Department of Physics and Astronomy, University of Sheffield, Hounsfield Road, Sheffield S3 7RH, United Kingdom

${ }^{4}$ Département de Physique, Université de Montréal, CP 6128 Succ. C-V, Montréal, QC, H3C 3J7, Canada

${ }^{5}$ Département de Physique, Université Laval, Pavillon Vachon, Quebec City, QC, G1K 7P4 Canada
} 
candidates, their properties and spatial distribution, and how we rule out most contaminants. The spatial distributions of the WR and RSG stars near a giant star-forming complex are strikingly different. WR stars dominate the complex core, while RSG dominate the complex halo. Future papers in this series will describe and catalog more than a thousand WR and RSG candidates that are detectable in our images, as well as spectra of many of those candidates.

Subject headings: galaxies: individual (M101) — galaxies: stellar content — stars: Wolf-Rayet — stars: Supergiants

\section{Introduction and Motivation}

\subsection{Starburst Regions}

The study of individual luminous stars and stellar populations in nearby giant H II regions is a prerequisite to understanding the starburst phenomenon and interpreting the observations of distant starburst galaxies and those containing starburst regions, for which only integral properties can be observed (Leitherer 1997). In this context, the Hubble Space Telescope (HST) has been crucial in providing us with high-resolution images of nearby, but very dense and massive, stellar clusters which are ionizing giant H II regions (Hunter et al. (1995), Hunter et al. (1996) and Malumuth et al. (1996)).

Our HST-based investigation of the stellar populations of the most luminous starforming complexes in the nearby late-type (ScIII) spiral galaxy NGC 2403 was very fruitful (Drissen et al. 1999), and underpinned our request for HST time to observe M101. A member of the M81 group, at a distance of $3.2 \pm 0.4 \mathrm{Mpc}$ (Freedman \& Madore 1988), NGC 2403 is very rich in H II regions (Sivan et al. 1990). Its abundance level and O/H radial gradient have been well established by Martin \& Belley (1996); they are similar to those of M33 (Henry \& Howard 1995). In contrast with M33, which contains relatively modest giant H II regions, four of NGC 2403's H II regions are exceptionally bright, with $\mathrm{H} \alpha$ luminosities $\mathrm{L}(H \alpha) \sim 0.8-1.5 \times 10^{40} \mathrm{erg} / \mathrm{s}$, comparable to the most massive starburst region in the Local Group, the 30 Doradus complex. We also found direct evidence for the presence of Wolf-Rayet (WR) stars in five of the six giant H II regions investigated; 25 - 40 WR stars are present in the NGC 2403-I giant H II region alone. HST has also provided optical and UV spectra of individual massive stars in NGC 1569 (Maoz et al. 2001), NGC 5398 (Sidoli et al. 2006) and NGC 925 (Adamo et al. 2011). Ground-based imagery and spectroscopy has revealed rich WR populations in M83 (Crowther et al. 2004) and NGC 5253 (Crowther et al. 1999). 
M101 (also known as NGC 5457 and the Pinwheel Galaxy) is the logical galaxy in which to extend this work. As the nearest giant grand design ScI spiral galaxy, it is brimming with H II regions, massive stars and at least 3,000 luminous star clusters (Barmby et al. 2006). About $6500 \mathrm{WR}$ stars are estimated to exist in the Milky Way (Shara et al. 1999); simplistically scaling up to the size and luminosity of M101 suggests a population of 1020,000 WR stars and even more Red Supergiants (RSGs). The star formation rate (SFR) in M101 (Lee et al. 2009) is probably a few times that of the Milky Way, and M101 is 50\% or more larger than our Galaxy. This, again, suggests a very substantial population of M101 WR stars. In addition, a rich treasury of (mostly) continuum imagery with HST was already in hand: over $135 \mathrm{ksec}$ of HST exposures. As described below, we used this database extensively to perform the image subtractions needed to isolate the strong emission-line WR stars and very red Red Supergiants from the other stellar populations.

\subsection{Wolf-Rayet and Red Supergiant Stars}

Wolf-Rayet (Crowther 2007) and Red Supergiant stars (Levesque (2010); Meynet et al. (2011)) are the massive stars that are easiest to identify in imaging surveys of galaxies because of their strong emission lines and extreme colors, respectively. They provide important constraints on the age of a starburst (Gazak et al. 2012) and on the mode of star formation (Crowther 2013). Single stars with initial masses $\left(\mathrm{M}_{i}>20 \mathrm{M}_{\odot}\right)$ are predicted to advance to the WR phase at approximately solar metallicity. WR stars possess strong stellar winds which produce a unique, emission-line spectrum displaying broad He II $\lambda 4686$ for hot nitrogen-rich (WN) and carbon-rich (WC) stars or C III $\lambda 4650+\mathrm{C}$ IV $\lambda 5808$ for (initially more massive) carbon-rich (WC) subtypes. WNh stars are a unique subclass of WR stars as these are luminous WN stars that are still burning hydrogen on the main sequence (de Koter et al. 1998), hence they are fundamentally different from their helium burning classical cousins.

Since the hydrogen-rich envelope has been removed from classical WN stars it follows that they are probably the progenitors of at least a subset of $\mathrm{H}$-poor Type Ib SN. Similarly, the removal of both the hydrogen and helium envelopes from WC stars should correspond to the absence of both these elements in the spectra of Type Ic SN. However, the WR-Type Ibc SN question remains unresolved as, to date, no direct detection of a Type Ib or Ic SN progenitor has been obtained. (Eldridge et al. 2013) have recently claimed that 12 SNIbc progenitors are invisible to as faint as absolute B, $\mathrm{V}$ and $\mathrm{R}$ magnitudes of -4 to -5 .

In contrast, RSG are predicted to arise from the evolution of less massive $\left(8-20 \mathrm{M}_{\odot}\right)$ stars and are therefore expected to appear later in the life of a starburst. Evolutionary models predict that single massive stars with $\mathrm{M}_{i} \sim 8-20 \mathrm{M}_{\odot}$ end their lives during the Red 
Supergiant (RSG) phase as H-rich Type II core-collapse supernovae (ccSNe). HST broadband pre-SN imaging has been able to confirm the RSG-Type II SN connection, particularly for SN 2003gd (Smartt et al. 2009). However, the highest mass RSG progenitor to date is only $\sim 16 \mathrm{M}_{\odot}($ Smartt 2009$)$, making these limits uncertain.

Models indicate that in instantaneous starbursts of low metallicity, these two populations are well separated in time, since only the most extreme stars $\left(M_{i} \geq 50 M_{\odot}\right)$ can shed enough mass to reach the WR stage. In regions of high metallicity, however, the simultaneous presence of WR and RSG stars can be expected for a short period of time, since lower mass stars $\left(\sim 25 \mathrm{M}_{\odot}\right)$ can also become WR after having spent some time as RSG (Maeder \& Mevnet 1994). WR and RSG are observed to coexist in the massive Galactic cluster Westerlund 1 (Clark et al. 2005). One of our key goals is to directly test this prediction in a single galaxy: M101. Kennicutt et al. (2003) have shown that in M101, over the galactocentric range 6-41 kpc, oxygen abundances are well fitted by an exponential distribution from approximately $1.3(\mathrm{O} / \mathrm{H})$ solar in the center to $1 / 15(\mathrm{O} / \mathrm{H})$ solar in the outermost regions. Equivalently, $\log \mathrm{O} / \mathrm{H}+12=8.8$ in the center of $\mathrm{M} 101$, and 7.5 in that galaxy's outer regions. Observing across the entire range of galactocentric distances in M101 (from 0 to $50 \mathrm{kpc}$ ) to measure how the absolute numbers and WR/RSG ratio changes across the galaxy is an equally important goal of our study.

\subsection{Star Clusters}

An early HST-based investigation by Drissen et al. (1993) targeted the WR population of M33 using narrow-band $\lambda 4686$ imaging surveys. NGC 604 is the largest giant H II region in the nearby star-forming galaxy M33 which lies at a distance of only $\mathrm{d} \sim 0.8 \mathrm{Mpc}$ (Scowcroft et al. 2009). Ground-based imaging with seeing 1.2" revealed that NGC 604 has a moderate WR population (Drissen et al. 1991). However this was significantly increased via high spatial resolution HST narrow-band imaging (Drissen et al. 1993), identifying the fainter WR population which corresponds to the lowest mass WR stars. At the core of each of the two most luminous giant H II regions in NGC 2403 lies a luminous, compact object (Drissen et al. 1999).

The discovery that very dense, massive star clusters form at the cores of all types of starbursts led to the suggestion that globular clusters were once located at the cores of massive starbursts (Meurer 1995; Whitmore \& Schweizer 1995; Ho \& Filippenko 1996a). The cases of HD 97950 (the ionizing core of NGC 3603 (Drissen et al.|1995)), R136 (Moffat et al. 1994) and (Crowther et al. 2010), NGC 2363 (Drissen et al. 2000), NGC 2403-I and NGC 2403-II (Drissen et al. 1999), M31 (Neugent et al. 2012) and M33 (Neugent \& Massey 2011) 
show that massive compact stellar clusters also form in more normal galaxies as well. How much more common are they in a very massive, actively star-forming galaxy like M101?

A striking feature in the NGC 2403 clusters is that RSG stars are mainly present over a more extended halo, while the young blue stars and most WR stars are in or close to a compact core. Stars more massive than $\sim 25 \mathrm{M}_{\odot}$ are not expected to go through a RSG phase before becoming WR stars. For $M_{i} \leq 20 \mathrm{M}_{\odot}$, stars evolve to RSG and explode as Type II supernovae without entering the WR phase. The timescale of these evolutionary paths is longer as one considers lower masses, hence the absence of RSG and presence of WR stars in the cores indicate that the population is dominated by very young and very massive stars. The presence of RSG in the halos signifies that we have an older mix of stars of various masses with $\mathrm{M} \gtrsim 8-15 \mathrm{M}_{\odot}$. The relative age spread and the spatial exclusion between RSG and WR stars are most obvious for the largest H II regions. Although of different ages, the proximity there of the WR and RSG stars suggests a triggering link between the two populations, which is a key part of this research program. WR stars and RSG are observed to co-exist in the Milky Way's most massive compact cluster Westerlund 1 (Clark et al. 2005). The luminosity, inferred mass and compact nature of Westerlund 1 are comparable with those of Super Star Clusters - previously identified only in external galaxies (see e.g. Bastian et al. (2012), Larsen et al. (2011) Whitmore et al. (2005) and Whitmore et al. (2011)).

\subsection{Supernova Environments}

Another key goal of this and related studies is obtaining narrow-band imaging of several nearby galaxies to produce a catalogue of $\sim 10^{4}$ WR stars. When a Type Ibc SN and/or gamma ray burst (Georgy et al. 2012) eventually occurs in one of these galaxies, our catalog should reveal the WR progenitor, confirming one of the strongest predictions of stellar evolutionary theory. We have obtained ground-based narrow-band imaging of several nearby star-forming galaxies (Bibby \& Crowther (2012), Bibby \& Crowther (2010) and Hadfield \& Crowther (2007)) and confirmed a subset of the WR candidates with multi-object spectroscopy. Given the average lifetime of a WR star of $\sim 0.3 \mathrm{Myr}$ (Crowther et al. 2003), we would expect one of the WR stars identified to produce a Type Ibc ccSN within the next few decades. Until then, we are able to compare the distribution of WR stars in their host galaxy with different types of SN to assess whether they represent a common population; and to check whether the predicted $\mathrm{WC} / \mathrm{WN}$ ratio varies across galaxies as predicted by theory.

Different ccSN are seen to be located in different regions of their host galaxies. For example, Type II ccSN follow the distribution of the host galaxy light whereas Type Ic 
SN are preferentially located in the brightest regions, similar to long Gamma-Ray Bursts (Fruchter et al. 2006). Furthermore, Type Ib and Ic SN were found to have different spatial distributions relative to the distribution of the host galaxy light, strongly suggesting that they have different progenitors (Kelly et al. 2008). If WN and WC stars are the progenitors of Type Ib and Ic SN, respectively, they should follow the same distribution as the corresponding supernovae. Indeed, Leloudas et al. (2010) applied this approach to spectroscopically confirmed WR stars in M83 (Hadfield et al. 2005) and found that WN and WC stars are located in different regions of their host galaxy. Moreover, the distribution of WN and WC stars are most consistent with those of Type Ib and Ic SNe, respectively. Given that this paper only concentrates on one region in M101, we postpone the discussion of our candidates' distribution until the next paper in this series.

The plan of this paper is as follows. In Section 2 we describe the narrowband imaging technique and our HST observations. The data reductions, including image processing, photometry and detection limits are presented in Section 3. Source selection from our images is described in Section 4 and we address the issue of contamination by variable stars in Section 5. Our methodology for locating Red Supergiants is presented in Section 6; we also compare the distributions of the WR and RSG candidates and star clusters in this section. We briefly summarize our results in Section 7.

\section{Techniques and Observations}

The need for targeted surveys that uniquely pick out WR stars is highlighted by Smartt et al. (2009) and by Eldridge et al. (2013), who discussed Type Ibc SN for which broad-band pre-SN imaging exists. Not one progenitor has been identified from these dozen SNe. This is because short exposure times did not allow the images to go deep enough to detect the continuum of a WR star. Had proper narrow-band surveys been available we would almost certainly, by now, have been able to provide strong evidence for the WR-SNIbc connection.

A powerful technique to detect individual Wolf-Rayet stars in crowded fields, such as the ones in M101, consists of subtracting a continuum image, normalized in both PSF and intensity, from an image obtained with a narrow-band filter centered on the He II $\lambda 4686$ emission line. One reason for this is that WR stars are much brighter in filters sensitive to their strong, broad emission lines, particularly He II $\lambda 4686$, than their continua, by up to 3 magnitudes (Massey \& Johnson 1998). Hence, WR stars can be easily identified from specific narrowband images but are difficult to detect in broad-band images. Moreover, WR stars detected in broad-band images alone cannot be distinguished from other blue supergiants. This 
narrowband-broadband technique has been successfully used on WFPC2 F469N images of giant H II regions in galaxies such as M33 (Drissen et al. 1993) and NGC 2403 (Drissen et al. 1999), detecting both isolated, individual WR stars and WR stars in unresolved clusters that include only a very small fraction of WR stars.

M101 lies at a distance of $6.4 \mathrm{Mpc}$ (Shappee \& Stanek 2011), almost twice as far as NGC 2403 (Freedman \& Madore 1988), so that $\sim 4 \times$ longer exposures are needed to reach similar magnitudes. In Cycle 17 we obtained HST/Wide Field Camera 3 (WFC3) pointings of 2 orbits per M101 field, under program ID 11635 (PI. Shara), with a total exposure time of 6106 seconds per field. This permitted us to image to a similar depth in M101 as we achieved in NGC 2403. We note that the systemic redshift of M101 $(+372 \mathrm{~km} / \mathrm{s})$ shifts the center of the He II $\lambda 4686$ emission line to $\lambda 4692 \AA$. This has virtually no effect on the detectability of WR stars in this galaxy, since the F469N filter transmission curve is fortuitously centered at $4693 \AA$, and the filter sensitivity is nearly constant from 4680 to $4710 \AA$, with a FWHM of $50 \AA$. It is true that the filter will capture essentially all HeII 4686 but exclude most NIII 4640, with only the red half of CIII 4650 included.

We used 18 pointings to cover the large majority of M101. Some gaps between CCD chips are inevitable. The fields covered are shown in Figure 1. The very different orientation of one of the pointings was necessary to provide a guide star for the observations, albeit resulting in overlap with another pointing. The coverage of our WFC3 images was selected based on the availability of deep archival continuum imaging. We used F435W, F555W and F814W ACS/WFC images, taken under program ID 9490 (PI. Kuntz) and ID 10918 (PI. Freedman), to represent continuum images so that only additional narrow-band F469N images were needed.

\section{Data Reductions}

Each WFC3 pointing was treated individually to ensure the best alignment with the ACS/WFC data and to make the size of each dataset more manageable. The corresponding ACS frames were drizzled with the WFC3 F469N pointing using the MULTIDRIzzLE task within IRAF1 to produce a coordinate system that was consistent between each different instrument and filter. Often small shifts and rotations were required to achieve the best alignment; these were calculated using GEOMAP. To achieve the best match, the WFC3 data

\footnotetext{
${ }^{1}$ IRAF is distributed by the National Optical Astronomy Observatory, which is operated by the Association of Universities for Research in Astronomy (AURA) under cooperative agreement with the National Science Foundation.
} 


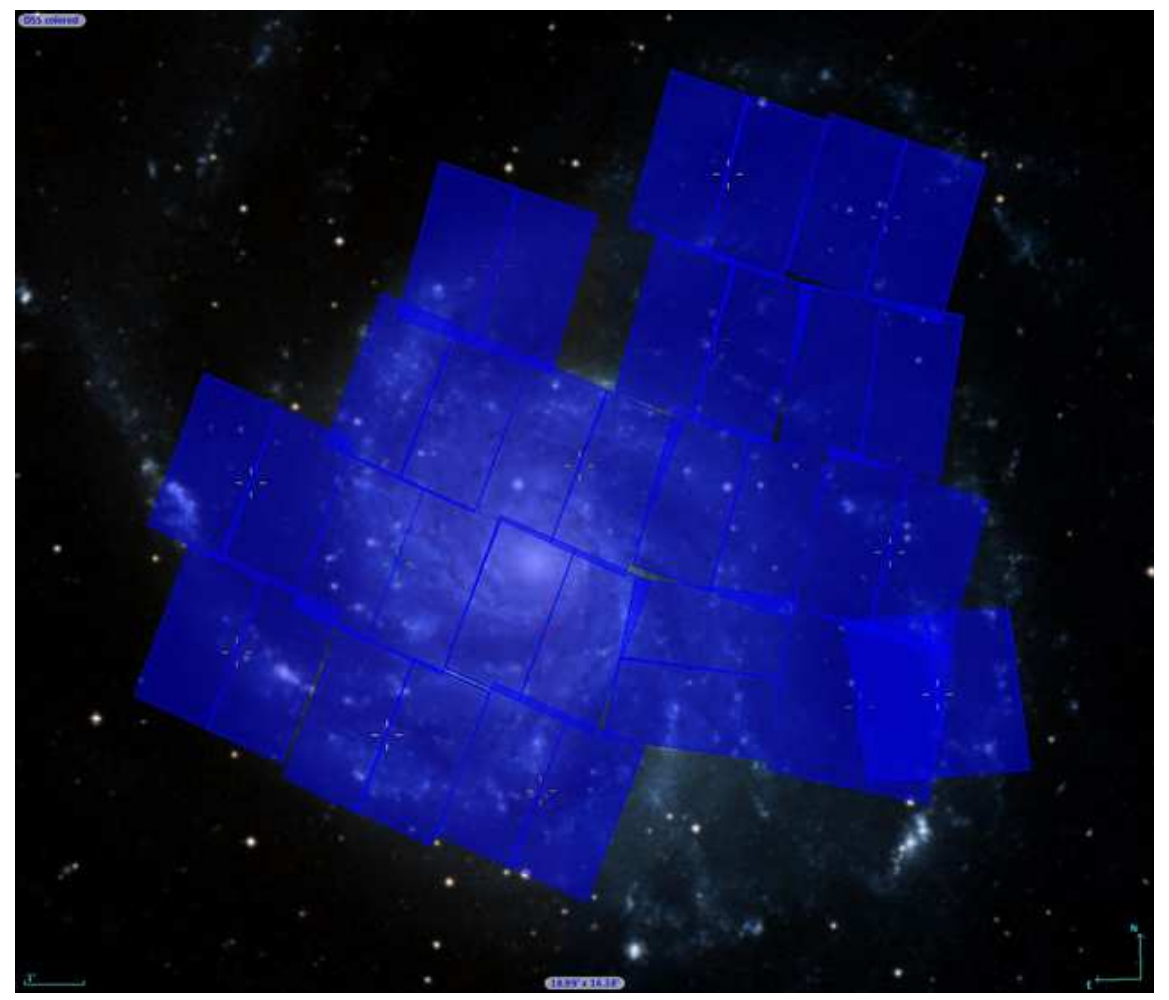

Fig. 1. - Digitized Sky Survey image of M101 with the 18 HST/WFC3 pointings overlaid. North is up and East to the left of the image. The field shown is approximately $20 \operatorname{arcmin}^{2}$ and each WFC3 field highlighted is $2.7 \times 2.7$ arcmin. 
was drizzled to a spatial scale of $0.15^{\prime \prime}$, corresponding to $4.65 \mathrm{pc}$ at the $6.4 \mathrm{Mpc}$ distance of M101. While slightly degraded from the $0.1^{\prime \prime}$ optimum sampling offered by HST, this was necessary to allow us to produce the best continuum subtractions possible.

In the following we describe the methods applied to all 18 pointings, and present the results of one of those pointings (M101-I). Later papers in this series, describing the remaining 17 fields, use exactly the same methodology.

\subsection{Photometry}

Once the broad- and narrow-band images had been aligned, photometry was performed on each filter separately using the standalone code, DAOPHOT (Stetson 1987). A point-spread function (PSF), based on isolated, point-like stars within the field, was built and applied to all the other stars detected. Individual zero-points from the HST literature were applied for each filter to transform the observed magnitudes into ST magnitudes and Vega magnitudes. For this study we find the Vega magnitude system to be more useful, since (under the Vega system) the F435W, F555W, and F814W filters correspond to the Johnson B, V and I filters. This enabled us to use literature color cuts. Henceforth, any magnitudes listed are Vega magnitudes unless otherwise stated.

Typical photometric errors for the narrow-band F469N images were \pm 0.07 mag for bright sources $\left(\mathrm{m}_{F 469 N}=21 \mathrm{mag}\right)$ and $\pm 0.5 \mathrm{mag}$ for the fainter sources $\left(\mathrm{m}_{F 469 N}=25.5 \mathrm{mag}\right)$; the distribution of the photometric errors relative to the source brightness for the F469N images is shown in Figure 2, For the ACS broad-band images the errors were slightly lower for sources of similar magnitudes, with $\pm 0.03 \mathrm{mag}\left(\mathrm{m}_{F 435 W}=21 \mathrm{mag}\right)$ and $\pm 0.10 \mathrm{mag}$ $\left(\mathrm{m}_{F 435 W}=25.5 \mathrm{mag}\right)$. For the faintest sources in the ACS image with $\mathrm{m}_{F 435 W}=28 \mathrm{mag}$ the photometric error is typically $\pm 0.5 \mathrm{mag}$, shown in Figure 3 for the F435W image, which is consistent with the other broad-band images.

\subsection{Detection Limits}

In order to assess the completeness of our M101 survey we must determine to what depth our images probe. Following the method of Bibby \& Crowther (2010) we fit a polynomial to the distribution, where the $100 \%$ detection limit is defined by the point at which the power law deviates from the observed data. Figure 4 shows the distribution of sources detected in our WFC3/F469N data, indicating that our $100 \%$ detection limit is $\mathrm{m}_{F 469 \mathrm{~N}}=24.3 \mathrm{mag}$. If we adopt the extinction from Lee et al. (2009) of $\mathrm{A}(\mathrm{H} \alpha)=1.06 \mathrm{mag}$, corresponding to 


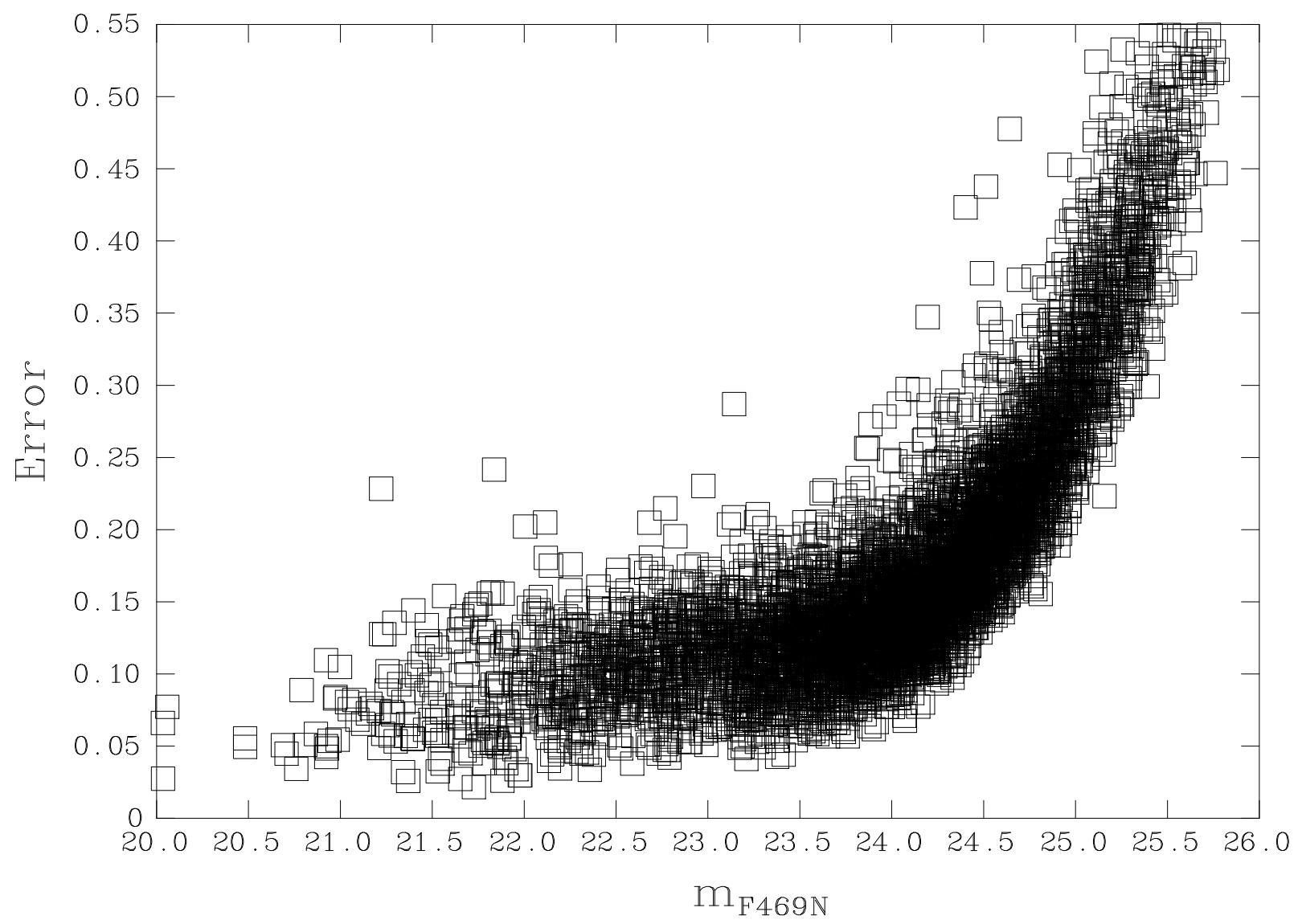

Fig. 2.- Photometric errors as a function of zero-point corrected, apparent (Vega) magnitude for all sources found in the WFC3/F469N image of M101-I . 


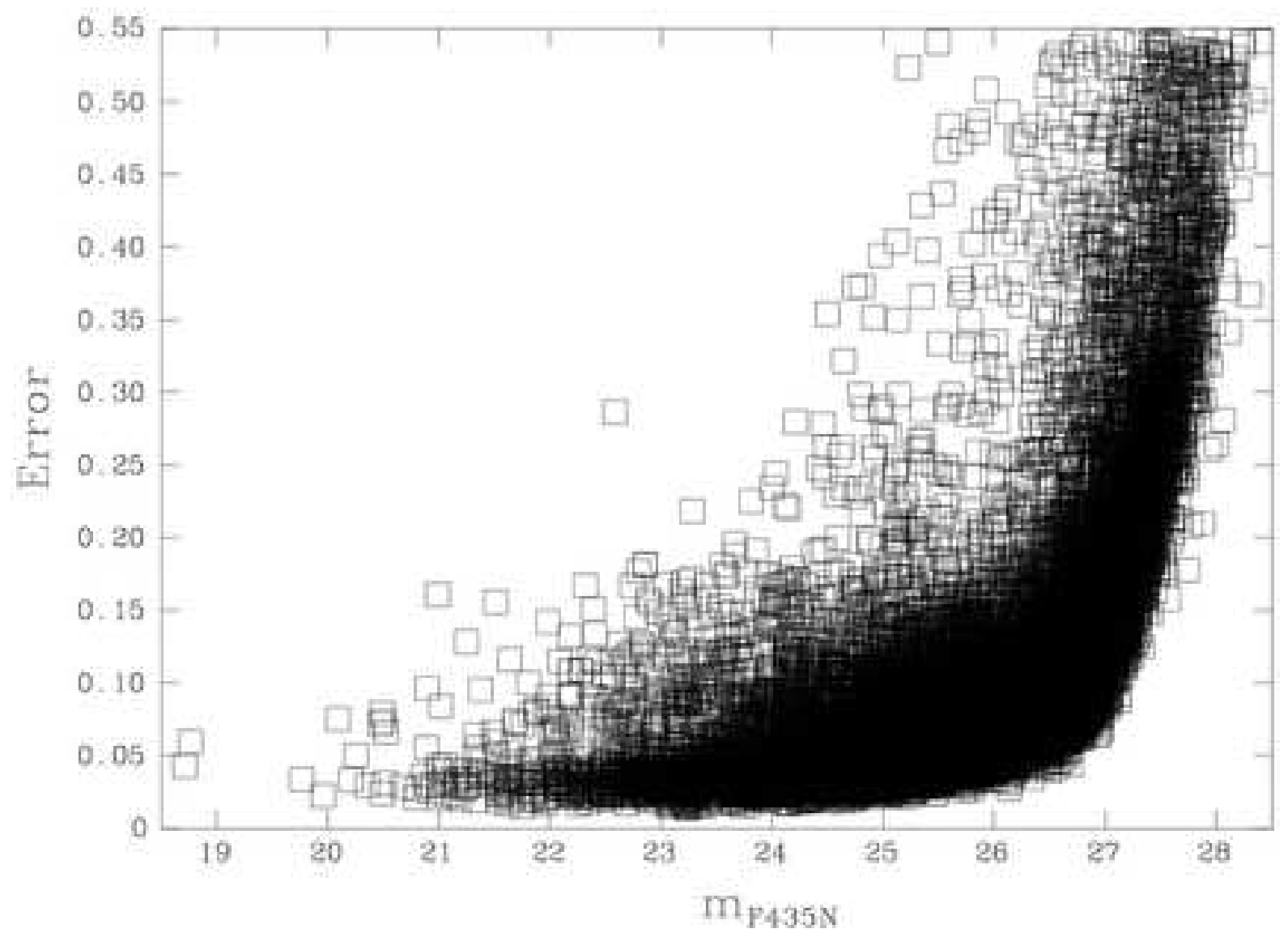

Fig. 3.- Photometric errors as a function of zero-point corrected, apparent (Vega) magnitude for all sources found in the ACS/WFC F435W image of M101-I . 
$\mathrm{A}(\mathrm{F} 469 \mathrm{~N})=1.53 \mathrm{mag}$ following the extinction law from Cardelli et al. (1989), and adopt the Cepheid distance of 6.4 Mpc (Shappee \& Stanek 2011), our 100\% completeness detection limit corresponds to $\mathrm{M}_{F 469 N}=-6.26$ mag.

The magnitude distribution of sources in the ACS/F435W data is shown in Figure 5 , which shows that we sample $\sim 2$ magnitudes fainter in the continuum, with a $100 \%$ detection limit of $\mathrm{m}_{F 435 W}=26.6 \mathrm{mag}$. Again, we adopt the extinction law of Cardelli et al. (1989) so $\mathrm{A}(\mathrm{F} 435 \mathrm{~W})=1.74 \mathrm{mag}$ determines a $100 \%$ completeness detection limit of $\mathrm{M}_{F 435 \mathrm{~W}}=-$ 4.17 mag.

\section{Source Selection}

The F469N filter, centered at $\lambda 4693 \AA$ includes the He II $\lambda 4686$ emission line, and partially includes the red wing of the CIII $\lambda 4650$ emission lines from carbon-rich WR stars, however the N III $\lambda 4640$ line from nitrogen-rich, WN, stars will not be detected. In order to identify WR candidates we had to identify sources which were brighter in the narrow-band image relative to the continuum image, in this case the F435W image centered at $\lambda 4297 \AA$. We note that we also tried creating a continuum image using a combination of the $\mathrm{F} 435 \mathrm{~W}$ and F555W images, however this did not improve our subtraction and hence we used the F435W and F555W images individually to check the accuracy of our candidates.

Output files from the photometric analysis were merged to match sources in terms of $\mathrm{x}$ and $\mathrm{y}$ coordinates. For each source $\mathrm{m}_{F 469 N^{-}} \mathrm{m}_{F 435 \mathrm{~W}}$ was determined, where $\mathrm{m}_{F 469 N^{-}}$ $\mathrm{m}_{F 435 W} \leq 0$ indicates an excess at $\lambda 4693 \AA$. Only sources where the excess was $\geq 3 \sigma$ were considered to be WR candidates. In addition, sources that were only identified in the F469N image, and not in the F435W or F555W images, were also flagged as WR candidates, since it is likely that these stars are faint WR stars with little or no detectable continuum. Figure 6] shows the photometric properties of each WR candidate, with the $100 \%$ detection limit of $\mathrm{m}_{F 435 \mathrm{~W}}=26.6 \mathrm{mag}$ adopted as the continum magnitude for those candidates only detected in the F469N image.

An efficient way of identifying bonafide WR candidates is via the "blinking" method (Moffat \& Shara 1983), which compares the F469N, F435W and continuum subtracted images in sequence. However, the broad-band F435W filter bandpass is almost $30 \times$ the width of the narrow-band F469N filter, hence the F435W image was scaled to create a narrow-band continuum. Since most stars should have $\mathrm{m}_{F 469 N}-\mathrm{m}_{F 435 W}=0$ we determined the scale factor which allowed most stars to disappear from the continuum subtracted image. We note that this could affect the detection of WR stars with a low He II excess. 


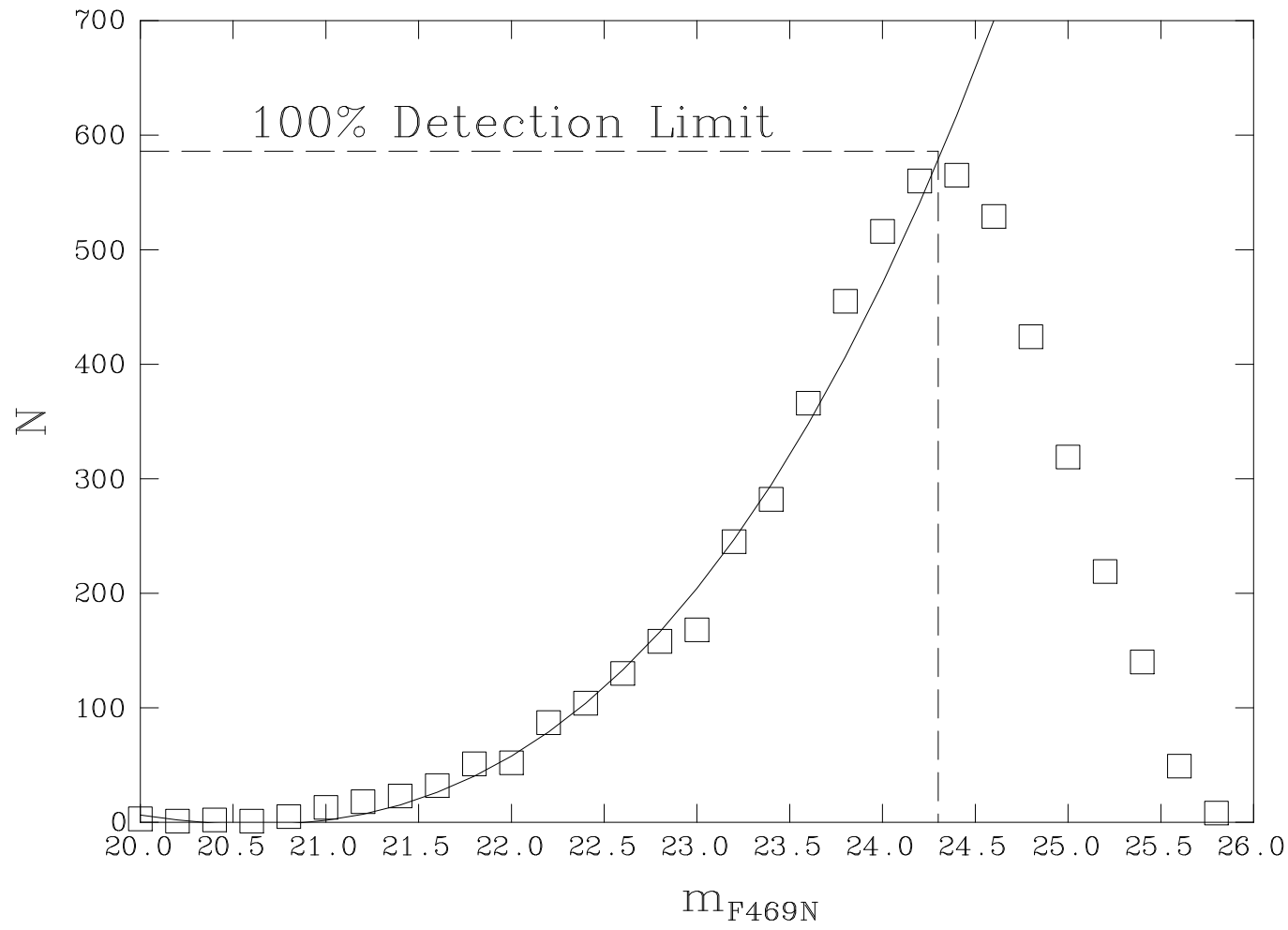

Fig. 4. - The magnitude distribution of photometric sources identified in the WFC3/F469N image of M101-I using 0.2 magnitude bins. A $100 \%$ detection limit of $\mathrm{m}_{F 469 \mathrm{~N}}=24.3 \mathrm{mag}$ is derived from this plot using the solid line which represents a 3rd degree polynomial fit to the brightest sources. 


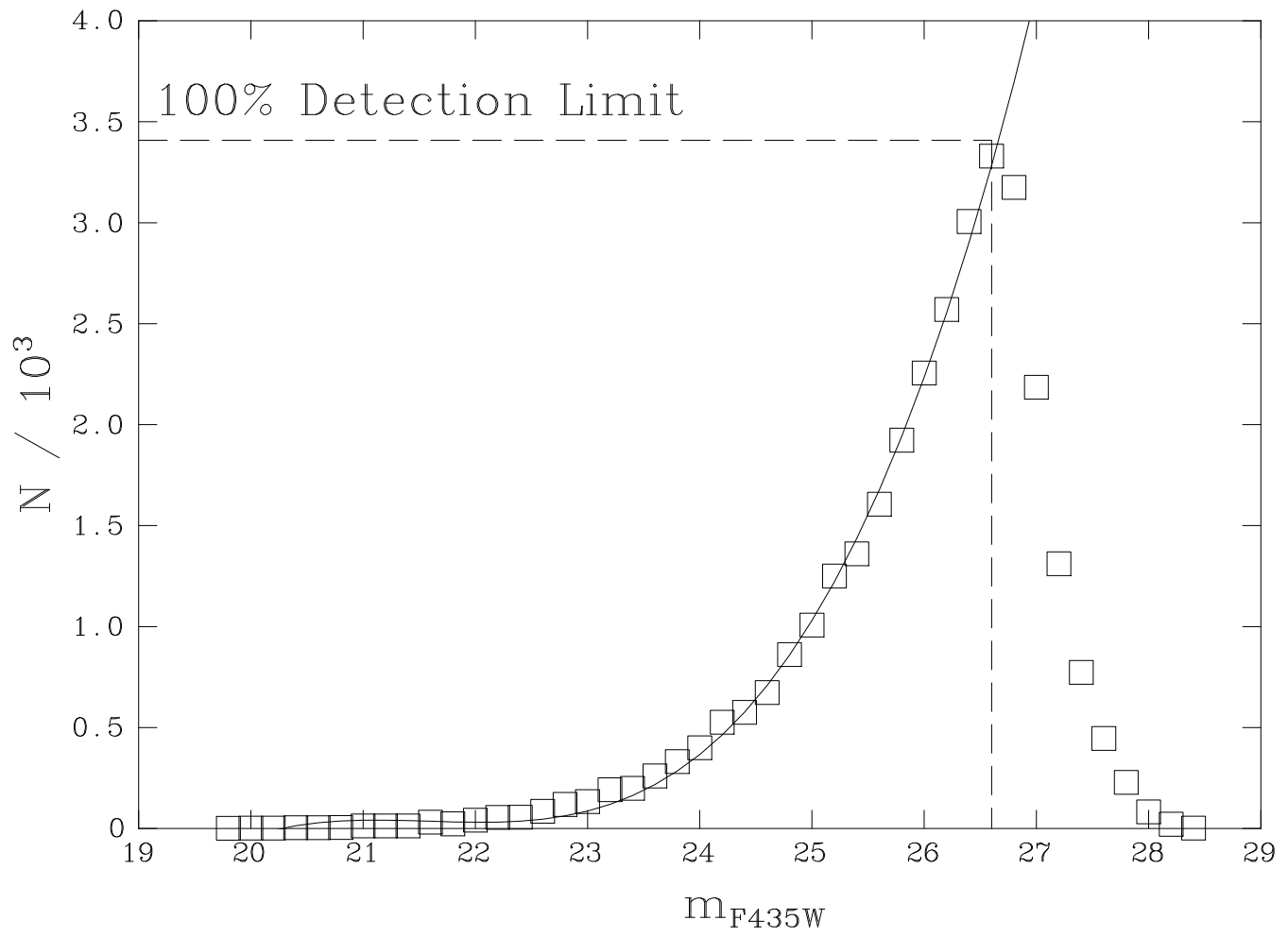

Fig. 5. - The magnitude distribution of photometric sources identified in the ACS/WFC F435W image of M101-I using 0.2 magnitude bins. A $100 \%$ detection limit of $\mathrm{m}_{F 435 \mathrm{~W}}=26.6 \mathrm{mag}$ is derived from this plot using the solid line which represents a 3rd degree polynomial fit to the brightest sources. 
The "blinking" technique was applied to all of the $3 \sigma$ photometric WR candidates to confirm the HeII $\lambda 4686$ excess and to remove any spurious sources such as cosmic rays, features at the edge of the CCD, and poor photometry in dense, unresolved regions.

In total, after the photometry and blinking processes, $91 \mathrm{WR}$ candidates were identified in just one of our F469N fields (M101-I). Of these, 54 candidates ( $\sim 60 \%)$ are detected only in the F469N image. Examples of typical candidates are shown in Figure 7 which shows the WR candidate in the F435W, F469N and F469N-F435W scaled $_{\text {filters. }}$

\section{Contamination by Variable Stars}

Since the narrow-band images were obtained in 2010, and the continuum images in 2002, it is possible that stellar variability over the 8 year baseline leads to contamination of our WR candidates with Cepheid and other variable stars.

Shappee \& Stanek (2011) identified Cepheids in 2 fields to determine the distance to M101. They showed that Cepheids have a (V-I) color $\geq 0.5 \mathrm{mag}$ (their Figure 12) which we can apply to our WR candidates. Of the $37 \mathrm{WR}$ candidates detected in both the F469N and F435W images, and identified to have a $\geq 3 \sigma$ excess in $\mathrm{m}_{F 469 N}-\mathrm{m}_{F 435 W}, 15$ are provisionally eliminated $(\sim 40 \%)$ by their red $(\mathrm{V}-\mathrm{I})>0.5 \mathrm{mag}$ colors. As already notes in Section 3.2 , reddening of $0.5 \mathrm{mag}$ of individual M101 stars, including WR stars, is expected. Hence some of these 15 stars with $(\mathrm{V}-\mathrm{I}) \geq 0.5 \mathrm{mag}$ colors may be reddened WR stars. However, to be conservative in our estimates we eliminate them for now. Two of the 22 surviving candidates were not detected in the $\mathrm{F} 814 \mathrm{~W}$ image, and two other candidates have a $\mathrm{V}-\mathrm{I}<-$ $1 \mathrm{mag}$; this is unexpected. However, inspection of the image reveals that these two sources lie in very crowded regions and hence their photometry is likely to be more unreliable than for the other candidates. The $(\mathrm{V}-\mathrm{I})$ colors for the 18 candidates which have a $\geq 3 \sigma$ excess and $-1<(\mathrm{V}-\mathrm{I})<0.5 \mathrm{mag}$ are shown in Figure 8, though we emphasize that 22 candidates are not eliminated by the above color test.

Unfortunately, for the WR candidates detected only in F469N, but not in F555W, we cannot calculate a V-I color. However, if we "blink" the F469N image with the F814W image we can identify the sources that are also detected in the F814W images. These stars are too red to be WR stars. Of our initial 54 candidates that were not detected in either F435W or F555W, only one is detected in the F814W image. It is is eliminated as a likely red star, reducing the number of WR candidates detected only in F469N, but not in F555W to 53 . In the M101-I region our final survey tally is $22+53=75$ candidate WR stars. Their photometry is presented in Table 1 . 


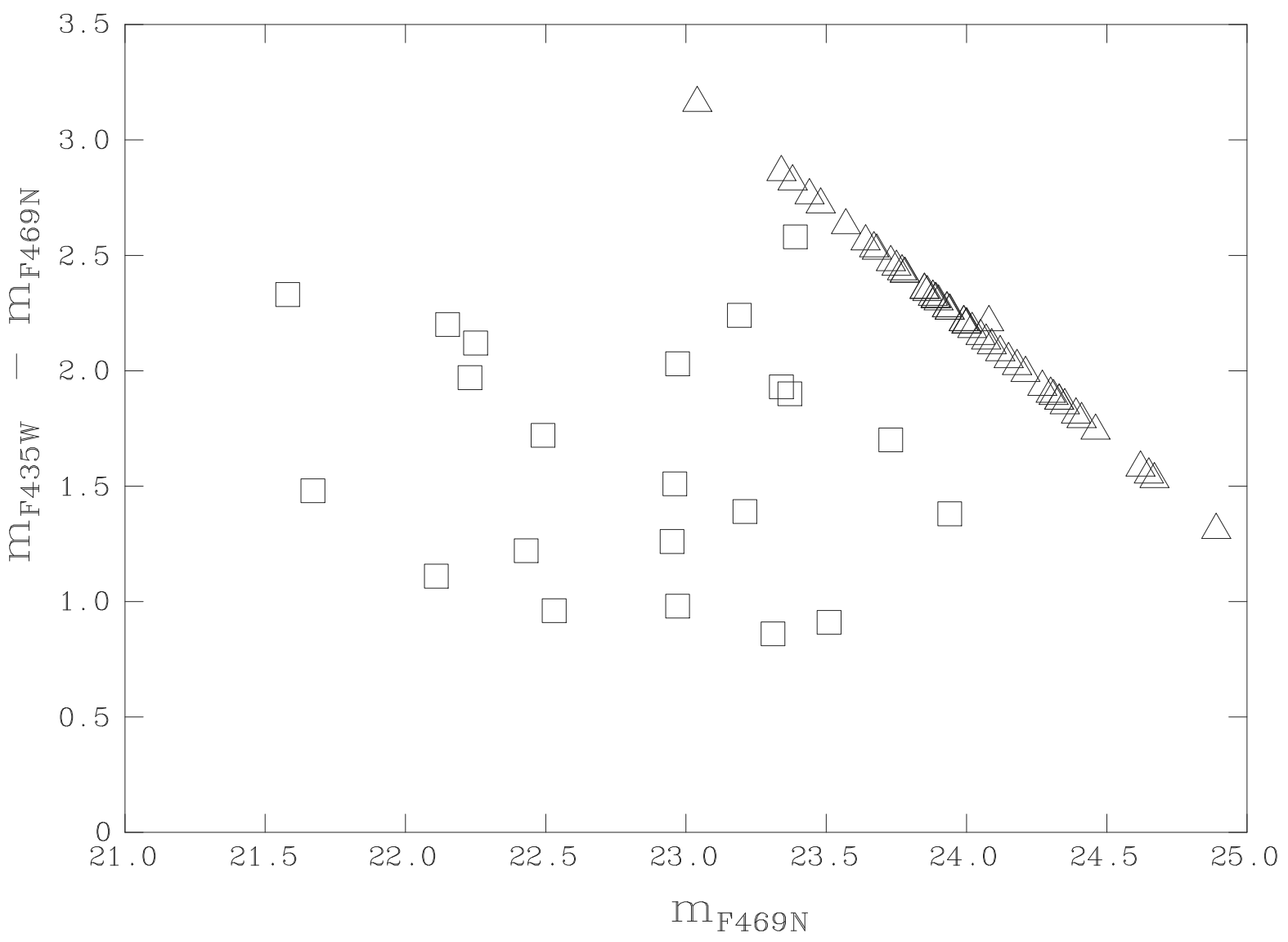

Fig. 6. - Excess, $\mathrm{m}_{F 435 W}-\mathrm{m}_{F 469 N}$, versus F469N magnitude for WR candidates in M101-I. Open squares indicate WR candidates with a $3 \sigma$ detection in both F469N and F435W images, which also have an excess of $>3 \sigma$. Open triangles indicate sources for which there was no detection in the $\mathrm{F} 435 \mathrm{~W}$ image, so excesses represent lower limits assuming $\mathrm{m}_{F 435 \mathrm{~W}}=26.6 \mathrm{mag}$ - our $100 \%$ detection limit. 


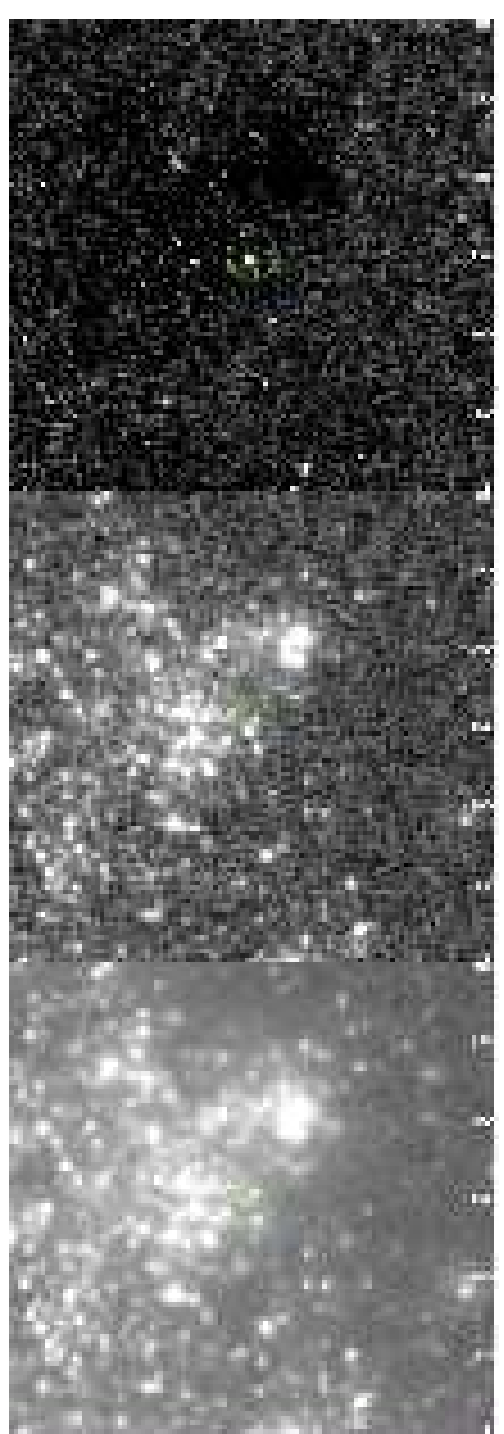

(a)

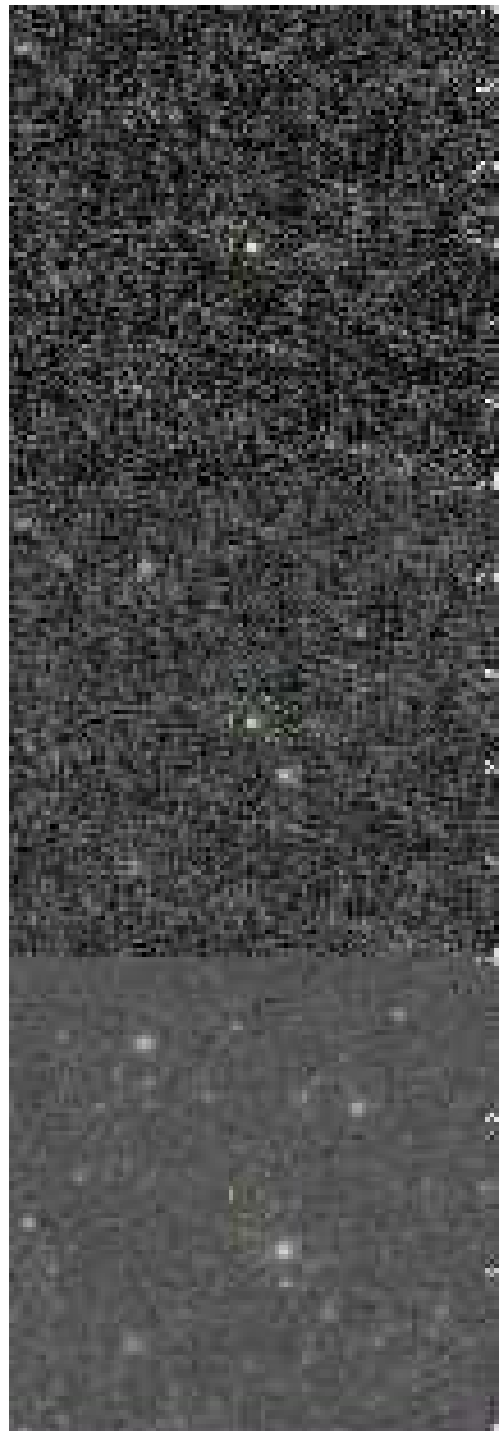

(b)

Fig. 7.- Postage stamp images produced during blinking showing the F435W continuum (bottom), F469N narrow-band (middle) and continuum subtracted image (top) for each WR candidate. Here we show an example of a WR candidate a) detected in the continuum (source \#1026), and b) not detected in the continuum image (source \#639). 
Blue variable stars could also be a source of contamination in our WR candidate list. We rule out $\mathrm{B}[\mathrm{e}]$ stars since they do not exhibit any emission lines that lie within the F469N filter bandpass. It is possible that we have identified a few Luminous Blue Variables (LBVs) during outburst, since the continuum of an LBV can vary by up to $\sim 2$ mag. We note that LBVs are rare, with only four confirmed in M33 (Clark et al. 2012). We expect few LBV to contaminate our WR candidate list, but an independent check, which follows, is prudent.

The HST archive has a rich assortment of M101 images, hence we used ACS/F555W images from 2006 (Proposal ID: 10918, PI Freedman), mid-way between the epochs of the ACS F435W (2002) and WFC3 F469N (2010) imaging, to investigate the impact of variable stars on our survey. The 2006 ACS/F555W imaging covers only a part of the M101-I frame covered by the 2002 narrowband data; 12 of our WR candidates lie within this region. The 2006 data were reduced and analyzed using the same method as described in Section 3 , again blinking in all available filters. 12 WR candidates were independently identified using the 2006 imaging, 11 of which are consistent with the analysis using the F555W imaging from 2002. The two remaining candidates, one from each epoch, are candidates that have been identified from the F469N emission and do not have a counterpart in the broad-band imaging. Both candidates are bonafide WR candidates and we conclude that they have most likely been missed through human error in the blinking process. The consistency and lack of variability of the WR candidates found in the two epochs again supports the view that variability is not a significant issue for our survey.

Finally we note that, after the analyses reported here were completed, we obtained Gemini-North spectra (which will be described in the next paper of this series) of over a hundred WR candidates in multiple fields; a high farction do, indeed, turn out to be bona fide WR stars.

\section{Identifying Red Supergiants}

The archival ACS images also allow us to identify RSG candidates from their $(\mathrm{B}-\mathrm{V})$ and $(\mathrm{V}-\mathrm{I})$ colors. Based on the colors of RSGs in the LMC we apply color cuts of $(\mathrm{B}-\mathrm{V}) \geq 1.2 \mathrm{mag}$ and $(\mathrm{V}-\mathrm{I}) \geq 1.8 \mathrm{mag}$ to our field of M101 (B. Davies, priv. communication). To ensure that we are not contaminated by foreground red giant stars we also insist that the luminosity of each RSG candidate is $\geq 10^{4.5} \mathrm{~L}_{\odot}$, equivalent to an apparent magnitude of $\mathrm{m}_{F 814 W} \leq 22 \mathrm{mag}$. In total we identify 164 RSG candidates in our single M101 field; their photometry is presented in Table 2 .

In their study of RSGs in M31, Massey et al. (2009) suggest a more stringent B-V cut of 


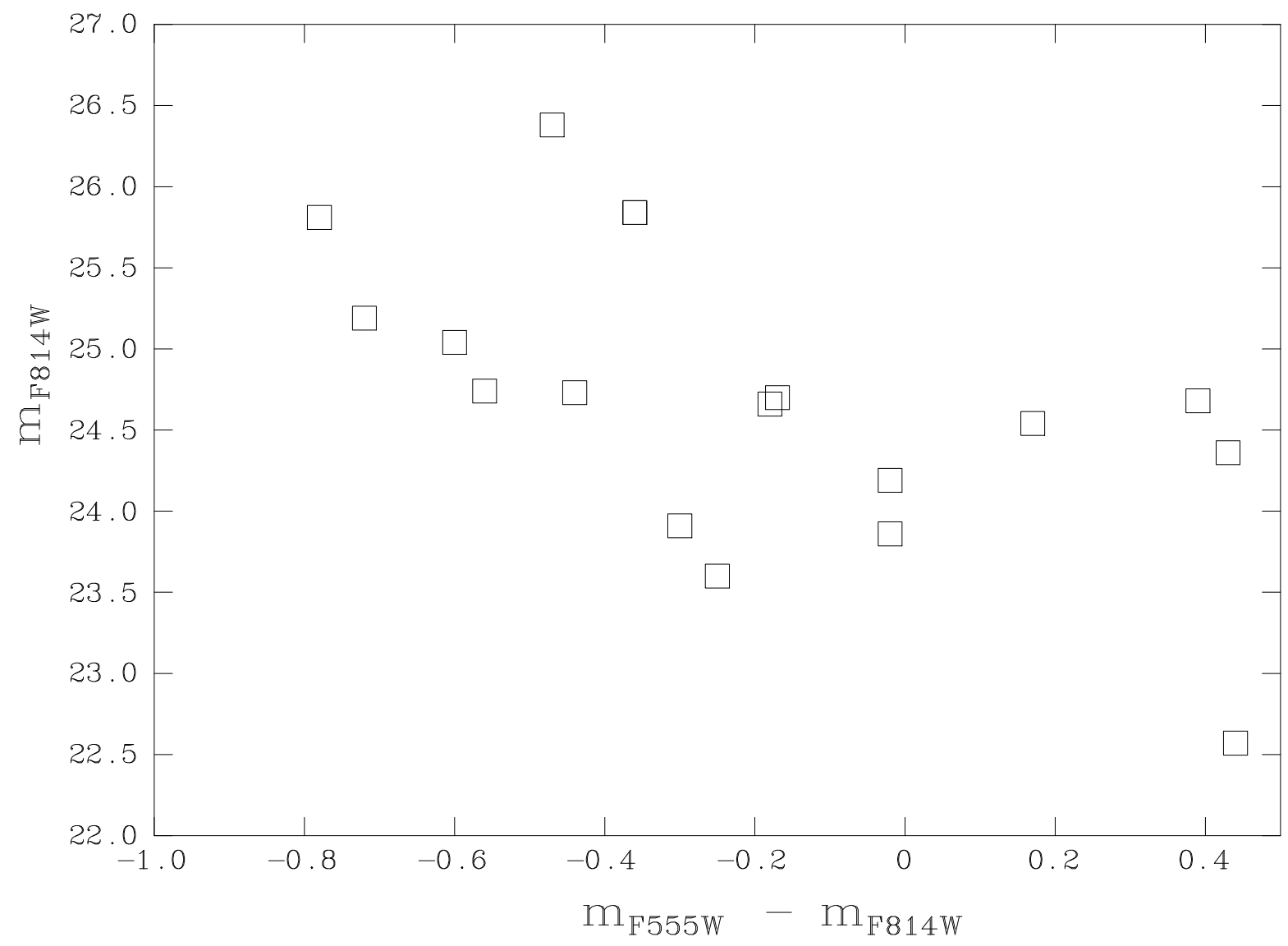

Fig. 8.- Here we present the I (F814W) versus (V-I) of the WR candidates in M101 which have a detection limit in both the F469N and F435W filter images of $>3 \sigma$. Following Shappee \& Stanek (2011) we use a color cut of V-I>0.5 mag to rule out contamination by Cepheids. This resulted in the rejection of 15 WR candidates. 
$\geq 1.6 \mathrm{mag}$ to remove contamination from foreground stars. However, as they note, adopting this cut removes $\sim 25 \%$ of RSGs already spectroscopically confirmed by Massey (1998) from their candidate list (their Figure 3 ). If $\mathrm{B}-\mathrm{V} \geq 1.6 \mathrm{mag}$ is applied to the LMC RSG sample then $\sim 30 \%$ of candidates would be missed, which is consistent with the $35 \%$ reduction in RSG candidates we find if we apply the B-V $\geq 1.6$ mag cut to our M101 sample. The V-I versus B-V colors for our RSG candidates and V-I versus I magnitude for our M101 RSGs are presented in Figure 9(a) and 9(b), respectively. These figures depict the candidates which would have been deleted by the more stringent B-V cut as solid squares and the "less stringent" candidates as open squares. Inspection of the open points in Figure 9(b) reveals that the photometry of $\sim 7 \mathrm{RSG}$ candidates appears to be inconsistent with the remaining candidates (with $\mathrm{m}_{F 814 W} \geq 20.3 \mathrm{mag}$ or $\mathrm{m}_{F 555 W}-\mathrm{m}_{F 814 W} \geq 3 \mathrm{mag}$ ). However, we note that the $\delta \mathrm{m}_{F 814 W}=3 \mathrm{mag}$ and $\delta \mathrm{m}_{F 555 W}-\mathrm{m}_{F 814 W}=2 \mathrm{mag}$ range is consistent with that found for the confirmed RSGs in the LMC and as such we do not remove the RSGs from the candidate list.

In Figure 10 we focus on the largest star-forming complex NGC 5462 in the field M101-I, which includes multiple bright H II regions from Hodge et al. (1990), H1159, 1169, 1170 and 1176. This region has also been studied by Chen et al. (2005) using HST images and for reference we show an archival $\mathrm{HST} / \mathrm{WFPC} 2 \mathrm{H} \alpha$ image of this region, marking the clusters identified by these authors.

RSG and WR candidates are plotted as red squares and red triangles, respectively. The brightest $1 \%$ of all pixels in the ACS field are colored blue, while the next brightest $4 \%$ of all pixels are colored black. 7 WR and 36 RSG candidates are "isolated", i.e. not surrounded by black pixels. $6 \mathrm{WR}$ and $30 \mathrm{RSG}$ are largely or entirely surrounded by black pixels. Finally, 12 WR and 5 RSG are surrounded by blue pixels. The corresponding ratios of numbers of WR to RSG candidates are 0.194, 0.2 and 2.4. The former two are statistically indistinguishable, but the strong clustering of WR stars in the core of the star-forming complex M101-I is suggested by the latter ratio. This suggestion can be made indisputable only with a much larger sample of stars. In later papers in this series we will greatly strengthen these small number statistics with thousands of M101 WR and RSG stars.

\section{Summary and Conclusions}

We describe the motivation for, and data collected by HST to search for the progenitors of type Ib/c supernovae in the nearby giant spiral galaxy M101. The analysis methodology and early results of a search for WR and RSG stars in one HST WFC3 pointing of M101 are reported. $75 \mathrm{WR}$ and $164 \mathrm{RSG}$ candidates are identified. There is a suggestion of 


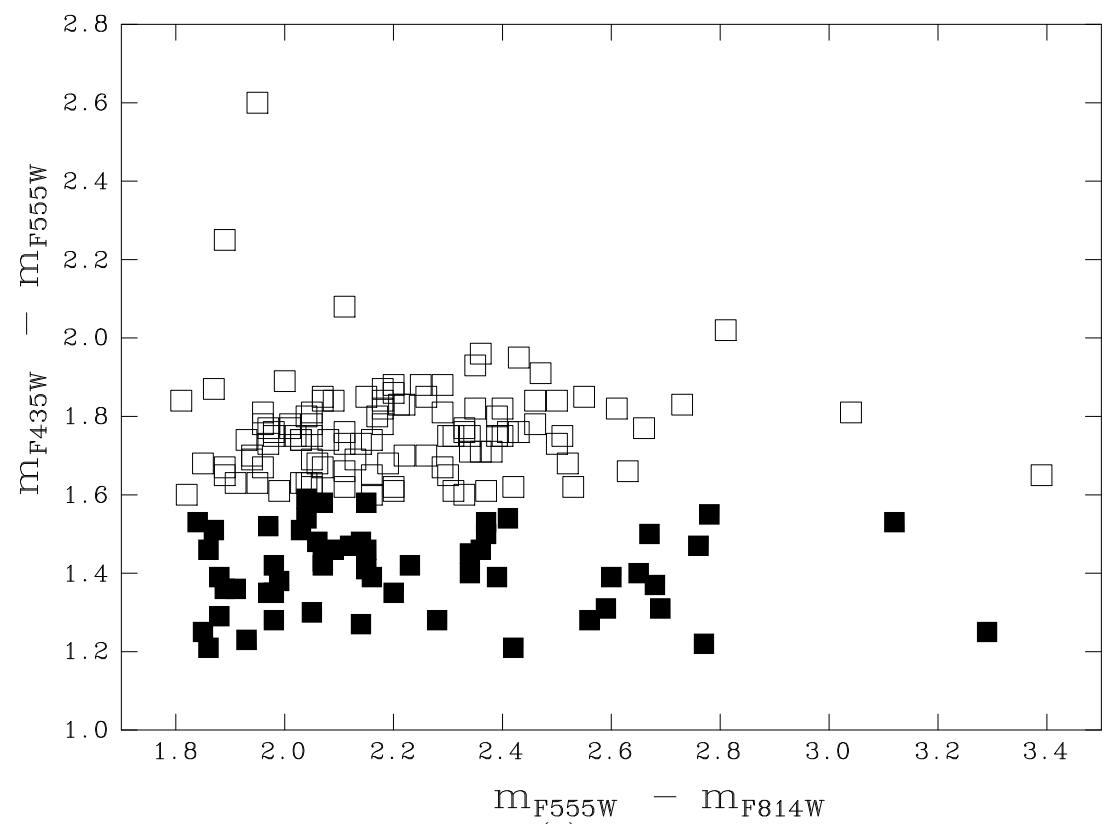

(a)

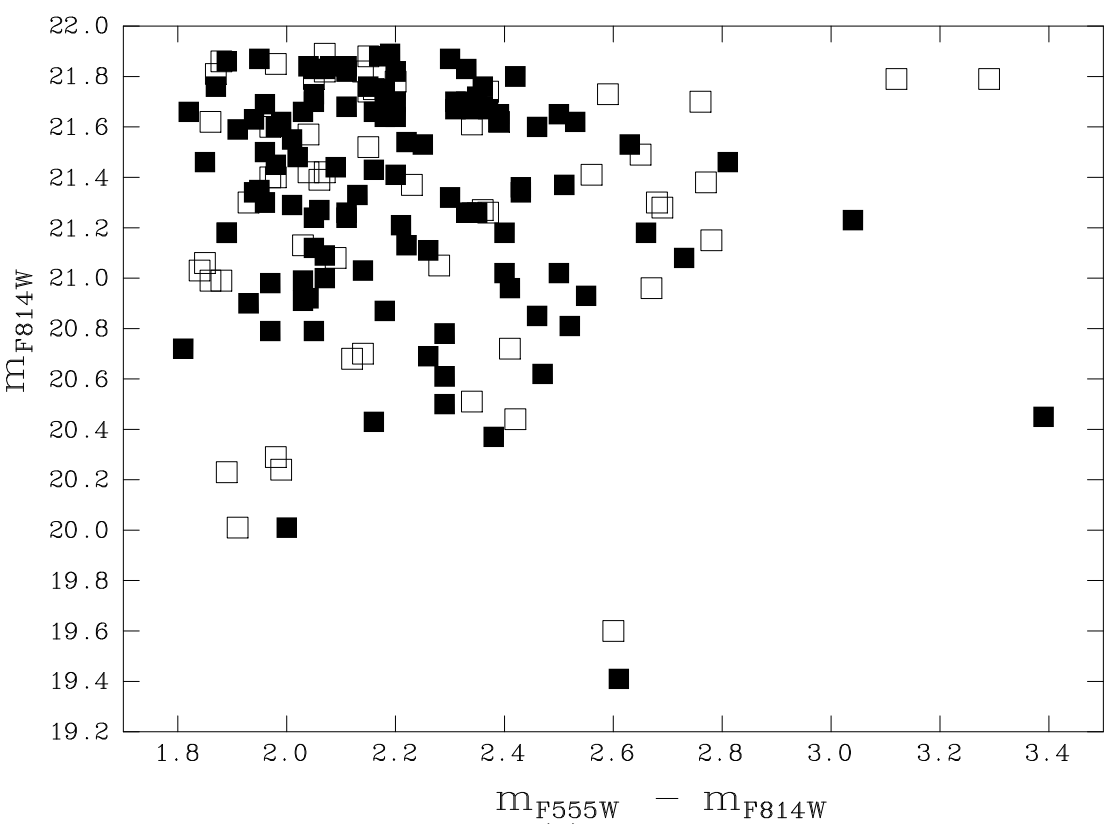

(b)

Fig. 9.- Photometry of Red Supergiant candidates in a single field of M101 showing a) the $\mathrm{V}-\mathrm{I}$ versus $\mathrm{B}-\mathrm{V}$ colors and b) $\mathrm{V}-\mathrm{I}$ versus I-band magnitude for all candidates. The solid squares show sources which are identified as RSGs using a $(B-V) \geq 1.2$ cut, while the open squares use the more stringent constraint of $(B-V) \geq 1.6$ mag from Massey et al. (2009). 


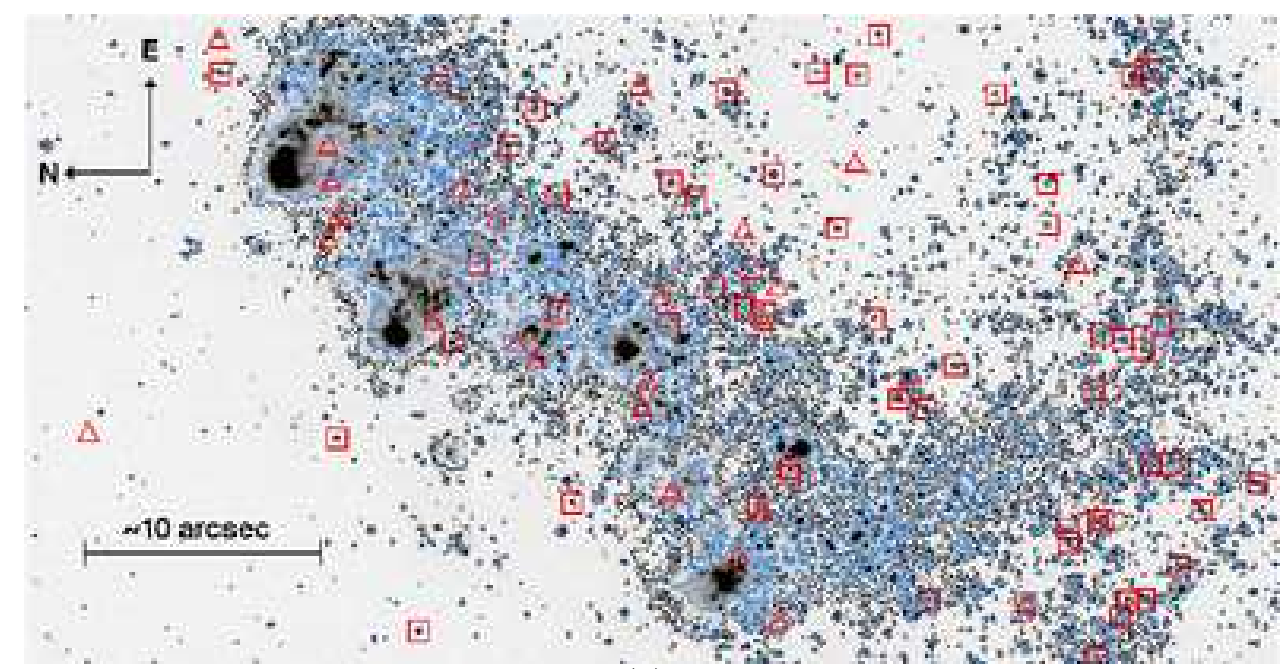

(a)

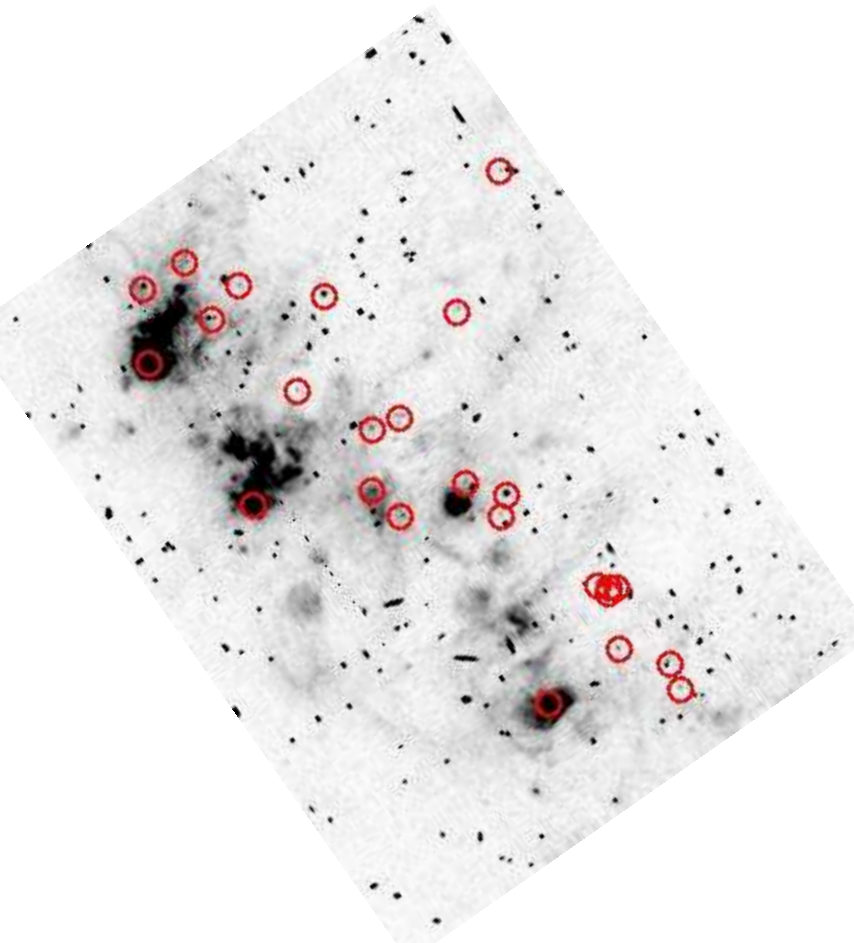

(b)

Fig. 10.- a) Broad band image of the main region of M101-I containing multiple clusters. The contours plotted related to $99 \%$ (blue) and 95\% (black) cuts for the brightness. WolfRayet stars are shown as red triangles and Red Supergiants as red squares - assuming a cut of $\mathrm{B}-\mathrm{V} \geq 1.6 \mathrm{mag}$ from Massey et al. (2009) and b) an archival WFPC2 H $\alpha$ image of the same region with clusters identified from Chen et al. (2005) plotted as red circles. 
Table 1: Photometry of M101 WR candidates. The RA and DEC of each candidate is taken from the calibration of the F469N/WFC3 image. Narrow- and broad-band magnitudes are listed for each candidate, unless the object was not detected in that filter. Errors listed are the $1 \sigma$ errors determined by the DAOPHOT routine.

\begin{tabular}{|c|c|c|c|c|c|c|c|c|c|c|c|}
\hline $\mathrm{RA}$ & DEC & F469N & err & F435W & err & F435W-F469N & err & F555W & err & F814W & err \\
\hline 14:03:51.802 & $+54: 21: 39.55$ & 22.11 & 0.07 & 23.22 & 0.01 & 1.11 & 0.07 & 23.61 & 0.03 & 23.91 & 0.05 \\
\hline 14:03:51.378 & $+54: 21: 35.21$ & 22.53 & 0.12 & 23.49 & 0.03 & 0.96 & 0.12 & 23.10 & 0.04 & 22.57 & 0.04 \\
\hline 14:03:51.722 & $+54: 21: 50.05$ & 23.21 & 0.13 & 24.60 & 0.04 & 1.39 & 0.13 & 24.71 & 0.04 & 24.54 & 0.05 \\
\hline 14:03:52.347 & $+54: 21: 54.57$ & 22.25 & 0.13 & 24.37 & 0.04 & 2.12 & 0.14 & 24.44 & 0.06 & 25.04 & 0.08 \\
\hline 14:03:52.755 & $+54: 21: 55.90$ & 23.37 & 0.09 & 25.27 & 0.05 & 1.90 & 0.10 & 25.48 & 0.08 & 25.84 & 0.11 \\
\hline 14:03:54.324 & $+54: 21: 55.99$ & 23.34 & 0.12 & 25.27 & 0.05 & 1.93 & 0.10 & 25.48 & 0.08 & 25.84 & 0.11 \\
\hline 14:03:53.820 & $+54: 22: 03.57$ & 23.94 & 0.14 & 25.32 & 0.05 & 1.38 & 0.15 & 25.07 & 0.06 & 24.68 & 0.07 \\
\hline 14:03:53.690 & $+54: 22: 08.78$ & 22.43 & 0.10 & 23.65 & 0.02 & 1.22 & 0.10 & 23.84 & 0.03 & 23.86 & 0.04 \\
\hline 14:03:53.863 & $+54: 22: 09.11$ & 21.58 & 0.12 & 23.91 & 0.04 & 2.33 & 0.13 & 24.18 & 0.06 & 24.74 & 0.07 \\
\hline 14:03:54.030 & $+54: 22: 09.25$ & 23.39 & 0.12 & 25.97 & 0.17 & 2.58 & 0.21 & 25.91 & 0.16 & 26.38 & 0.52 \\
\hline 14:03:53.344 & $+54: 21: 50.08$ & 23.73 & 0.11 & 25.43 & 0.05 & 1.70 & 0.12 & 25.96 & 0.08 & - & - \\
\hline 14:03:53.172 & $+54: 21: 50.63$ & 23.19 & 0.12 & 25.43 & 0.05 & 2.24 & 0.12 & 25.96 & 0.08 & - & - \\
\hline 14:03:52.987 & $+54: 22: 00.44$ & 21.67 & 0.10 & 23.15 & 0.09 & 1.48 & 0.13 & 23.35 & 0.10 & 23.60 & 0.11 \\
\hline 14:03:53.072 & $+54: 22: 01.25$ & 22.15 & 0.14 & 24.35 & 0.05 & 2.20 & 0.14 & 24.47 & 0.04 & 25.19 & 0.08 \\
\hline 14:03:53.676 & $+54: 22: 02.08$ & 22.23 & 0.14 & 24.20 & 0.03 & 1.97 & 0.14 & 24.10 & 0.03 & 25.28 & 0.07 \\
\hline 14:03:53.294 & $+54: 21: 55.20$ & 22.97 & 0.13 & 23.95 & 0.04 & 0.98 & 0.13 & 24.17 & 0.04 & 24.19 & 0.04 \\
\hline 14:03:54.942 & $+54: 22: 03.39$ & 23.51 & 0.17 & 24.42 & 0.04 & 0.91 & 0.17 & 24.53 & 0.05 & 24.70 & 0.07 \\
\hline 14:03:53.180 & $+54: 22: 04.81$ & 22.49 & 0.17 & 24.21 & 0.06 & 1.72 & 0.18 & 24.29 & 0.08 & 25.49 & 0.20 \\
\hline 14:03:54.810 & $+54: 22: 08.67$ & 22.95 & 0.10 & 24.21 & 0.06 & 1.26 & 0.18 & 24.29 & 0.08 & 24.73 & 0.13 \\
\hline 14:03:54.551 & $+54: 22: 13.94$ & 22.97 & 0.15 & 25.00 & 0.05 & 2.03 & 0.16 & 25.03 & 0.07 & 25.81 & 0.10 \\
\hline 14:03:36.863 & $+54: 23: 00.19$ & 22.96 & 0.14 & 24.47 & 0.04 & 1.51 & 0.15 & 24.79 & 0.05 & 24.36 & 0.04 \\
\hline 14:03:53.453 & $+54: 21: 37.31$ & 23.31 & 0.17 & 24.17 & 0.03 & 0.86 & 0.17 & 24.48 & 0.02 & 24.66 & 0.03 \\
\hline 14:03:52.091 & $+54: 21: 27.92$ & 24.08 & 0.17 & - & - & - & - & - & - & - & - \\
\hline 14:03:44.772 & $+54: 21: 38.30$ & 23.93 & 0.17 & - & - & - & - & - & - & - & - \\
\hline 14:03:47.409 & $+54: 21: 40.92$ & 23.99 & 0.18 & - & - & - & - & - & - & - & - \\
\hline 14:03:53.946 & $+54: 21: 46.79$ & 24.21 & 0.14 & - & - & - & - & - & - & - & - \\
\hline 14:03:43.399 & $+54: 22: 10.12$ & 24.41 & 0.20 & - & - & - & - & - & - & - & - \\
\hline 14:03:46.325 & $+54: 22: 24.73$ & 23.88 & 0.11 & - & - & - & - & - & - & - & - \\
\hline 14:03:49.713 & $+54: 23: 06.55$ & 23.75 & 0.12 & - & - & - & - & - & - & - & - \\
\hline 14:03:45.544 & $+54: 23: 10.90$ & 23.78 & 0.15 & - & - & - & - & - & - & - & - \\
\hline 14:03:48.384 & $+54: 23: 21.04$ & 23.94 & 0.10 & - & - & - & - & - & - & - & - \\
\hline 14:03:45.830 & $+54: 23: 23.92$ & 24.05 & 0.12 & - & - & - & - & - & - & - & - \\
\hline
\end{tabular}


Table 1: (continued)

\begin{tabular}{|c|c|c|c|c|c|c|c|c|c|c|c|}
\hline RA & DEC & F469N & err & $\overline{\mathrm{F} 435 \mathrm{~W}}$ & err & F435W-F469N & err & F555W & err & $\overline{\text { F814W }}$ & $\overline{\text { err }}$ \\
\hline 14:03:43.572 & $+54: 20: 43.93$ & 24.15 & 0.17 & - & - & - & - & - & - & - & - \\
\hline $14: 03: 42.956$ & $+54: 20: 56.05$ & 23.94 & 0.17 & - & - & - & - & - & - & - & - \\
\hline 14:03:43.450 & $+54: 21: 03.90$ & 24.33 & 0.12 & - & - & - & - & - & - & - & - \\
\hline $14: 03: 42.025$ & $+54: 21: 38.77$ & 23.38 & 0.13 & - & - & - & - & - & - & - & - \\
\hline 14:03:52.247 & $+54: 21: 50.81$ & 23.89 & 0.13 & - & - & - & - & - & - & - & - \\
\hline $14: 03: 53.618$ & $+54: 21: 51.54$ & 23.68 & 0.11 & - & - & - & - & - & - & - & - \\
\hline 14:03:47.278 & $+54: 21: 52.04$ & 23.67 & 0.18 & - & - & - & - & - & - & - & - \\
\hline 14:03:51.110 & $+54: 22: 05.84$ & 24.00 & 0.12 & - & - & - & - & - & - & - & - \\
\hline 14:03:53.577 & $+54: 22: 09.21$ & 23.93 & 0.18 & - & - & - & - & - & - & - & - \\
\hline 14:03:43.080 & $+54: 22: 17.27$ & 23.99 & 0.11 & - & - & - & - & - & - & - & - \\
\hline $14: 03: 46.935$ & $+54: 22: 18.13$ & 24.33 & 0.16 & - & - & - & - & - & - & - & - \\
\hline 14:03:48.954 & $+54: 22: 18.41$ & 23.85 & 0.13 & - & - & - & - & - & - & - & - \\
\hline 14:03:52.630 & $+54: 22: 19.51$ & 23.73 & 0.15 & - & - & - & - & - & - & - & - \\
\hline 14:03:50.926 & $+54: 22: 31.61$ & 23.86 & 0.15 & - & - & - & - & - & - & - & - \\
\hline 14:03:46.177 & $+54: 22: 33.89$ & 23.34 & 0.11 & - & - & - & - & - & - & - & - \\
\hline 14:03:36.468 & $+54: 22: 40.98$ & 24.46 & 0.19 & - & - & - & - & - & - & - & - \\
\hline 14:03:51.363 & $+54: 22: 42.89$ & 24.27 & 0.23 & - & - & - & - & - & - & - & - \\
\hline 14:03:39.703 & $+54: 23: 07.76$ & 23.89 & 0.14 & - & - & - & - & - & - & - & - \\
\hline 14:03:43.267 & $+54: 23: 10.18$ & 23.64 & 0.11 & - & - & - & - & - & - & - & - \\
\hline 14:03:48.790 & $+54: 23: 30.75$ & 23.90 & 0.10 & - & - & - & - & - & - & - & - \\
\hline 14:03:46.573 & $+54: 20: 58.59$ & 24.09 & 0.19 & - & - & - & - & - & - & - & - \\
\hline 14:03:40.353 & $+54: 21: 10.67$ & 24.31 & 0.30 & - & - & - & - & - & - & - & - \\
\hline 14:03:51.245 & $+54: 21: 17.95$ & 23.77 & 0.14 & - & - & - & - & - & - & - & - \\
\hline 14:03:47.905 & $+54: 21: 19.20$ & 24.00 & 0.18 & - & - & - & - & - & - & - & - \\
\hline 14:03:45.569 & $+54: 22: 03.20$ & 24.02 & 0.16 & - & - & - & - & - & - & - & - \\
\hline 14:03:53.393 & $+54: 22: 22.78$ & 23.48 & 0.17 & - & - & - & - & - & - & - & - \\
\hline 14:03:35.495 & $+54: 22: 50.76$ & 23.89 & 0.18 & - & - & - & - & - & - & - & - \\
\hline 14:03:43.473 & $+54: 23: 19.11$ & 24.89 & 0.31 & - & - & - & - & - & - & - & - \\
\hline 14:03:40.107 & $+54: 21: 14.66$ & 23.85 & 0.26 & - & - & - & - & - & - & - & - \\
\hline 14:03:44.015 & $+54: 21: 48.57$ & 24.30 & 0.20 & - & - & - & - & - & - & - & - \\
\hline 14:03:40.020 & $+54: 21: 49.87$ & 24.62 & 0.48 & - & - & - & - & - & - & - & - \\
\hline 14:03:49.533 & $+54: 21: 57.22$ & 24.18 & 0.35 & - & - & - & - & - & - & - & - \\
\hline 14:03:46.586 & $+54: 22: 44.52$ & 23.78 & 0.13 & - & - & - & - & - & - & - & - \\
\hline $14: 03: 43.833$ & $+54: 23: 10.16$ & 24.00 & 0.15 & - & - & - & - & - & - & - & - \\
\hline 14:03:48.099 & $+54: 23: 39.66$ & 24.67 & 0.26 & - & - & - & - & - & - & - & - \\
\hline 14:03:45.029 & $+54: 21: 01.14$ & 23.57 & 0.20 & - & - & - & - & - & - & - & - \\
\hline 14:03:52.031 & $+54: 21: 51.80$ & 23.04 & 0.12 & - & - & - & - & - & - & - & - \\
\hline $14: 03: 52.886$ & $+54: 21: 55.63$ & 23.44 & 0.14 & - & - & - & - & - & - & - & - \\
\hline 14:03:50.001 & $+54: 23: 19.95$ & 24.07 & 0.30 & - & - & - & - & - & - & - & - \\
\hline 14:03:40.677 & $+54: 22: 19.23$ & 24.65 & 0.20 & - & - & - & - & - & - & - & - \\
\hline 14:03:43.142 & $+54: 22: 34.54$ & 24.39 & 0.19 & - & - & - & - & - & - & - & - \\
\hline 14:03:43.161 & $+54: 22: 33.92$ & 24.35 & 0.18 & - & - & - & - & - & - & - & - \\
\hline 14:03:38.669 & $+54: 22: 18.74$ & 24.12 & 0.16 & - & - & - & - & - & - & - & - \\
\hline
\end{tabular}


Table 2: HST/ACS photometry of Red Supergiant candidates in one pointing of M101. In total we identify 164 RSG candidates using color and magnitude cuts provided by B. Davies (priv. communication). Note that all magnitudes presented use the Vega magnitude system, for which zero points were provided by Josh Sokol from the ACS instrument team. Errors listed represent $1 \sigma$ errors which are calculated in DAOРнOT.

\begin{tabular}{|c|c|c|c|c|c|c|c|}
\hline RA & DEC & F435W & err & F555W & err & $\mathrm{F} 814 \mathrm{~W}$ & err \\
\hline 14:03:53.731 & $+54: 21: 53.65$ & 25.40 & 0.05 & 24.02 & 0.03 & 21.62 & 0.05 \\
\hline 14:03:51.361 & $+54: 21: 13.24$ & 25.40 & 0.05 & 23.95 & 0.04 & 21.61 & 0.05 \\
\hline 14:03:52.891 & $+54: 21: 45.49$ & 24.94 & 0.04 & 23.48 & 0.02 & 21.62 & 0.03 \\
\hline 14:03:51.615 & $+54: 21: 35.75$ & 24.93 & 0.08 & 23.58 & 0.03 & 21.60 & 0.03 \\
\hline 14:03:52.696 & $+54: 21: 38.56$ & 25.63 & 0.08 & 24.32 & 0.04 & 21.73 & 0.05 \\
\hline 14:03:52.978 & $+54: 21: 45.75$ & 25.35 & 0.04 & 23.89 & 0.03 & 21.74 & 0.04 \\
\hline 14:03:55.206 & $+54: 21: 54.40$ & 25.65 & 0.04 & 24.12 & 0.03 & 21.74 & 0.04 \\
\hline 14:03:36.509 & $+54: 22: 49.55$ & 25.30 & 0.05 & 23.91 & 0.04 & 21.75 & 0.05 \\
\hline 14:03:51.397 & $+54: 22: 46.20$ & 25.33 & 0.05 & 23.98 & 0.03 & 21.78 & 0.06 \\
\hline 14:03:53.121 & $+54: 21: 27.84$ & 25.14 & 0.07 & 23.84 & 0.03 & 21.79 & 0.04 \\
\hline 14:03:35.420 & $+54: 21: 11.50$ & 26.33 & 0.08 & 25.08 & 0.07 & 21.79 & 0.08 \\
\hline 14:03:53.275 & $+54: 21: 21.52$ & 26.43 & 0.07 & 24.91 & 0.05 & 21.79 & 0.06 \\
\hline 14:03:52.172 & $+54: 21: 47.34$ & 25.19 & 0.07 & 23.68 & 0.04 & 21.81 & 0.06 \\
\hline 14:03:36.375 & $+54: 20: 48.33$ & 25.23 & 0.04 & 23.96 & 0.03 & 21.82 & 0.04 \\
\hline 14:03:54.280 & $+54: 21: 32.75$ & 25.32 & 0.07 & 23.89 & 0.04 & 21.82 & 0.05 \\
\hline 14:03:53.141 & $+54: 21: 47.40$ & 25.25 & 0.07 & 23.84 & 0.03 & 21.85 & 0.04 \\
\hline 14:03:53.387 & $+54: 21: 27.42$ & 25.03 & 0.10 & 23.73 & 0.05 & 21.86 & 0.06 \\
\hline 14:03:56.414 & $+54: 22: 32.07$ & 25.43 & 0.08 & 24.03 & 0.05 & 21.88 & 0.06 \\
\hline 14:03:51.467 & $+54: 21: 14.10$ & 25.38 & 0.05 & 23.96 & 0.05 & 21.89 & 0.06 \\
\hline 14:03:42.430 & $+54: 23: 36.30$ & 25.15 & 0.03 & 23.61 & 0.02 & 21.57 & 0.04 \\
\hline 14:03:52.154 & $+54: 21: 39.26$ & 25.25 & 0.07 & 23.67 & 0.04 & 21.52 & 0.05 \\
\hline 14:03:51.011 & $+54: 21: 08.24$ & 25.94 & 0.08 & 24.46 & 0.04 & 21.70 & 0.05 \\
\hline 14:03:54.974 & $+54: 21: 53.05$ & 25.53 & 0.07 & 24.14 & 0.05 & 21.49 & 0.05 \\
\hline 14:03:53.114 & $+54: 21: 47.13$ & 25.06 & 0.04 & 23.46 & 0.03 & 21.42 & 0.03 \\
\hline 14:03:52.144 & $+54: 21: 38.98$ & 25.07 & 0.07 & 23.49 & 0.03 & 21.42 & 0.04 \\
\hline 14:03:34.066 & $+54: 22: 47.59$ & 25.25 & 0.03 & 23.97 & 0.03 & 21.41 & 0.04 \\
\hline 14:03:52.364 & $+54: 21: 34.34$ & 24.93 & 0.07 & 23.45 & 0.02 & 21.39 & 0.03 \\
\hline 14:03:52.121 & $+54: 21: 41.35$ & 24.65 & 0.04 & 23.37 & 0.03 & 21.40 & 0.04 \\
\hline 14:03:41.571 & $+54: 23: 52.62$ & 24.89 & 0.04 & 23.37 & 0.03 & 21.40 & 0.05 \\
\hline 14:03:36.366 & $+54: 23: 07.19$ & 25.37 & 0.07 & 24.15 & 0.06 & 21.38 & 0.09 \\
\hline 14:03:51.947 & $+54: 21: 47.81$ & 25.02 & 0.12 & 23.60 & 0.05 & 21.37 & 0.06 \\
\hline 14:03:51.641 & $+54: 21: 37.61$ & 24.62 & 0.10 & 23.17 & 0.02 & 21.08 & 0.04 \\
\hline 14:03:54.354 & $+54: 21: 33.96$ & 24.45 & 0.06 & 23.23 & 0.03 & 21.30 & 0.04 \\
\hline 14:03:54.335 & $+54: 21: 33.92$ & 25.35 & 0.07 & 23.98 & 0.04 & 21.30 & 0.05 \\
\hline 14:03:45.593 & $+54: 22: 35.95$ & 25.09 & 0.11 & 23.63 & 0.11 & 21.27 & 0.16 \\
\hline 14:03:52.214 & $+54: 21: 28.58$ & 25.27 & 0.04 & 23.96 & 0.06 & 21.28 & 0.07 \\
\hline 14:03:50.700 & $+54: 21: 17.50$ & 25.13 & 0.06 & 23.63 & 0.06 & 21.26 & 0.07 \\
\hline 14:03:51.731 & $+54: 21: 37.81$ & 25.49 & 0.04 & 23.94 & 0.02 & 21.15 & 0.02 \\
\hline
\end{tabular}


Table 2: (continued)

\begin{tabular}{|c|c|c|c|c|c|c|c|}
\hline $\mathrm{RA}$ & DEC & F435W & err & F555W & err & F814W & err \\
\hline 14:03:51.696 & $+54: 21: 18.03$ & 24.66 & 0.05 & 23.15 & 0.06 & 21.13 & 0.09 \\
\hline 14:03:52.707 & $+54: 21: 48.57$ & 24.40 & 0.06 & 22.87 & 0.05 & 21.03 & 0.06 \\
\hline 14:03:51.869 & $+54: 21: 33.31$ & 24.61 & 0.04 & 23.34 & 0.12 & 21.05 & 0.13 \\
\hline 14:03:54.266 & $+54: 22: 09.33$ & 24.16 & 0.06 & 22.91 & 0.04 & 21.06 & 0.06 \\
\hline 14:03:52.993 & $+54: 21: 54.36$ & 24.06 & 0.10 & 22.85 & 0.10 & 20.99 & 0.10 \\
\hline 14:03:36.424 & $+54: 21: 20.63$ & 24.25 & 0.03 & 22.87 & 0.03 & 20.99 & 0.04 \\
\hline 14:03:53.214 & $+54: 21: 58.09$ & 24.32 & 0.07 & 22.84 & 0.05 & 20.70 & 0.06 \\
\hline 14:03:53.119 & $+54: 21: 40.82$ & 24.67 & 0.03 & 23.13 & 0.02 & 20.72 & 0.03 \\
\hline 14:03:51.525 & $+54: 21: 21.18$ & 24.27 & 0.02 & 22.80 & 0.02 & 20.68 & 0.03 \\
\hline 14:03:51.344 & $+54: 21: 00.17$ & 24.25 & 0.09 & 22.85 & 0.06 & 20.51 & 0.07 \\
\hline 14:03:52.993 & $+54: 21: 54.36$ & 24.06 & 0.10 & 22.85 & 0.10 & 20.44 & 0.10 \\
\hline 14:03:35.731 & $+54: 22: 49.13$ & 23.59 & 0.03 & 22.20 & 0.03 & 19.60 & 0.04 \\
\hline 14:03:53.537 & $+54: 21: 59.31$ & 23.27 & 0.08 & 21.91 & 0.03 & 20.01 & 0.04 \\
\hline 14:03:53.403 & $+54: 21: 59.38$ & 23.48 & 0.05 & 22.12 & 0.03 & 20.23 & 0.05 \\
\hline 14:03:54.224 & $+54: 21: 51.88$ & 23.61 & 0.06 & 22.23 & 0.03 & 20.24 & 0.04 \\
\hline 14:03:52.916 & $+54: 21: 50.23$ & 23.63 & 0.03 & 22.28 & 0.03 & 20.29 & 0.05 \\
\hline 14:03:56.477 & $+54: 22: 11.98$ & 23.84 & 0.03 & 22.02 & 0.04 & 19.41 & 0.05 \\
\hline 14:03:56.795 & $+54: 21: 54.97$ & 23.90 & 0.03 & 22.02 & 0.03 & 20.01 & 0.04 \\
\hline 14:03:41.464 & $+54: 23: 35.21$ & 24.46 & 0.05 & 22.75 & 0.03 & 20.37 & 0.04 \\
\hline 14:03:52.410 & $+54: 21: 49.64$ & 24.34 & 0.09 & 22.60 & 0.03 & 20.43 & 0.04 \\
\hline 14:03:44.033 & $+54: 23: 05.94$ & 25.49 & 0.05 & 23.84 & 0.03 & 20.45 & 0.04 \\
\hline 14:03:52.476 & $+54: 21: 34.12$ & 24.68 & 0.03 & 22.80 & 0.02 & 20.50 & 0.03 \\
\hline 14:03:52.475 & $+54: 21: 33.35$ & 24.57 & 0.04 & 22.90 & 0.04 & 20.61 & 0.04 \\
\hline 14:03:54.025 & $+54: 22: 01.54$ & 25.00 & 0.06 & 23.09 & 0.04 & 20.62 & 0.05 \\
\hline 14:03:52.267 & $+54: 21: 50.93$ & 24.64 & 0.07 & 22.94 & 0.03 & 20.69 & 0.05 \\
\hline 14:03:53.067 & $+54: 22: 03.94$ & 24.36 & 0.04 & 22.53 & 0.03 & 20.72 & 0.04 \\
\hline 14:03:55.499 & $+54: 21: 35.99$ & 24.88 & 0.04 & 23.07 & 0.03 & 20.78 & 0.04 \\
\hline 14:03:53.845 & $+54: 21: 54.61$ & 24.53 & 0.05 & 22.84 & 0.03 & 20.79 & 0.04 \\
\hline 14:03:54.357 & $+54: 21: 34.98$ & 24.48 & 0.05 & 22.76 & 0.04 & 20.79 & 0.06 \\
\hline 14:03:51.656 & $+54: 22: 05.41$ & 25.01 & 0.05 & 23.33 & 0.03 & 20.81 & 0.05 \\
\hline 14:03:53.157 & $+54: 21: 54.75$ & 25.15 & 0.10 & 23.31 & 0.09 & 20.85 & 0.13 \\
\hline 14:03:53.882 & $+54: 21: 50.27$ & 24.90 & 0.03 & 23.06 & 0.02 & 20.87 & 0.03 \\
\hline 14:03:52.740 & $+54: 21: 43.79$ & 24.57 & 0.03 & 22.83 & 0.03 & 20.90 & 0.03 \\
\hline 14:03:52.598 & $+54: 22: 08.88$ & 24.68 & 0.04 & 22.94 & 0.03 & 20.91 & 0.03 \\
\hline 14:03:51.297 & $+54: 21: 19.64$ & 24.76 & 0.05 & 22.96 & 0.04 & 20.92 & 0.05 \\
\hline 14:03:36.903 & $+54: 22: 09.95$ & 25.33 & 0.04 & 23.49 & 0.04 & 20.93 & 0.05 \\
\hline 14:03:50.700 & $+54: 21: 17.50$ & 25.13 & 0.06 & 23.37 & 0.04 & 20.96 & 0.05 \\
\hline 14:03:51.813 & $+54: 21: 34.43$ & 24.72 & 0.04 & 22.95 & 0.03 & 20.98 & 0.04 \\
\hline 14:03:52.838 & $+54: 21: 36.65$ & 24.76 & 0.05 & 23.02 & 0.03 & 20.99 & 0.04 \\
\hline 14:03:53.105 & $+54: 21: 36.27$ & 24.93 & 0.03 & 23.08 & 0.02 & 21.00 & 0.02 \\
\hline 14:03:54.367 & $+54: 21: 46.68$ & 25.23 & 0.04 & 23.42 & 0.02 & 21.02 & 0.03 \\
\hline 14:03:50.964 & $+54: 21: 07.60$ & 25.37 & 0.06 & 23.52 & 0.03 & 21.02 & 0.04 \\
\hline
\end{tabular}


Table 2: (continued)

\begin{tabular}{|c|c|c|c|c|c|c|c|}
\hline $\mathrm{RA}$ & DEC & F435W & err & F555W & err & $\overline{\mathrm{F} 814 \mathrm{~W}}$ & err \\
\hline 14:03:56.166 & $+54: 21: 59.42$ & 24.90 & 0.04 & 23.17 & 0.04 & 21.03 & 0.05 \\
\hline 14:03:51.522 & $+54: 21: 36.49$ & 25.64 & 0.05 & 23.81 & 0.04 & 21.08 & 0.04 \\
\hline 14:03:54.866 & $+54: 21: 54.63$ & 25.00 & 0.05 & 23.16 & 0.03 & 21.09 & 0.04 \\
\hline 14:03:52.377 & $+54: 21: 29.64$ & 25.22 & 0.03 & 23.37 & 0.04 & 21.11 & 0.05 \\
\hline 14:03:53.444 & $+54: 21: 14.05$ & 24.97 & 0.04 & 23.17 & 0.03 & 21.12 & 0.05 \\
\hline 14:03:51.733 & $+54: 21: 35.50$ & 25.18 & 0.07 & 23.35 & 0.09 & 21.13 & 0.12 \\
\hline $14: 03: 52.200$ & $+54: 21: 36.24$ & 25.33 & 0.07 & 23.07 & 0.03 & 21.18 & 0.04 \\
\hline 14:03:52.195 & $+54: 21: 36.42$ & 24.74 & 0.03 & 23.07 & 0.03 & 21.18 & 0.04 \\
\hline 14:03:52.744 & $+54: 21: 20.43$ & 25.61 & 0.05 & 23.84 & 0.04 & 21.18 & 0.05 \\
\hline 14:03:51.808 & $+54: 21: 35.15$ & 25.24 & 0.03 & 23.42 & 0.02 & 21.21 & 0.03 \\
\hline 14:03:51.182 & $+54: 21: 57.21$ & 26.08 & 0.07 & 24.27 & 0.03 & 21.23 & 0.05 \\
\hline 14:03:54.198 & $+54: 22: 00.43$ & 24.92 & 0.03 & 23.29 & 0.02 & 21.24 & 0.03 \\
\hline 14:03:53.621 & $+54: 21: 47.58$ & 25.09 & 0.04 & 23.36 & 0.03 & 21.24 & 0.04 \\
\hline 14:03:54.963 & $+54: 21: 55.03$ & 25.34 & 0.04 & 23.60 & 0.03 & 21.26 & 0.04 \\
\hline 14:03:54.378 & $+54: 22: 13.81$ & 25.54 & 0.05 & 23.61 & 0.03 & 21.26 & 0.04 \\
\hline 14:03:50.700 & $+54: 21: 17.50$ & 25.13 & 0.06 & 23.37 & 0.04 & 21.26 & 0.06 \\
\hline 14:03:54.288 & $+54: 21: 52.26$ & 25.00 & 0.06 & 23.32 & 0.02 & 21.27 & 0.03 \\
\hline 14:03:51.808 & $+54: 21: 43.68$ & 25.08 & 0.06 & 23.31 & 0.02 & 21.29 & 0.02 \\
\hline 14:03:42.360 & $+54: 23: 48.05$ & 25.04 & 0.08 & 23.26 & 0.06 & 21.30 & 0.08 \\
\hline 14:03:44.006 & $+54: 23: 36.89$ & 25.37 & 0.10 & 23.62 & 0.12 & 21.32 & 0.18 \\
\hline 14:03:55.025 & $+54: 22: 27.55$ & 25.15 & 0.06 & 23.46 & 0.03 & 21.33 & 0.04 \\
\hline 14:03:51.405 & $+54: 21: 41.58$ & 24.98 & 0.03 & 23.28 & 0.01 & 21.34 & 0.02 \\
\hline 14:03:52.443 & $+54: 21: 23.97$ & 25.73 & 0.06 & 23.77 & 0.04 & 21.34 & 0.05 \\
\hline 14:03:54.388 & $+54: 21: 34.39$ & 25.89 & 0.07 & 23.30 & 0.03 & 21.35 & 0.04 \\
\hline 14:03:52.154 & $+54: 21: 37.76$ & 25.54 & 0.05 & 23.78 & 0.03 & 21.36 & 0.04 \\
\hline 14:03:38.802 & $+54: 21: 47.99$ & 25.63 & 0.06 & 23.88 & 0.03 & 21.37 & 0.04 \\
\hline 14:03:56.427 & $+54: 22: 04.82$ & 25.48 & 0.05 & 23.60 & 0.03 & 21.41 & 0.05 \\
\hline 14:03:54.025 & $+54: 21: 42.82$ & 25.19 & 0.04 & 23.59 & 0.03 & 21.43 & 0.04 \\
\hline 14:03:50.921 & $+54: 21: 22.55$ & 25.37 & 0.05 & 23.53 & 0.03 & 21.44 & 0.04 \\
\hline 14:03:52.789 & $+54: 21: 44.94$ & 25.20 & 0.03 & 23.44 & 0.01 & 21.45 & 0.02 \\
\hline 14:03:53.838 & $+54: 21: 38.62$ & 26.29 & 0.08 & 24.27 & 0.03 & 21.46 & 0.03 \\
\hline 14:03:52.447 & $+54: 21: 49.52$ & 24.99 & 0.06 & 23.31 & 0.05 & 21.46 & 0.06 \\
\hline 14:03:54.564 & $+54: 21: 19.97$ & 25.25 & 0.05 & 23.50 & 0.02 & 21.48 & 0.07 \\
\hline 14:03:53.208 & $+54: 21: 50.69$ & 25.27 & 0.05 & 23.46 & 0.03 & 21.50 & 0.05 \\
\hline 14:03:54.040 & $+54: 21: 57.44$ & 25.67 & 0.06 & 23.78 & 0.03 & 21.53 & 0.04 \\
\hline 14:03:53.151 & $+54: 21: 33.80$ & 25.82 & 0.05 & 24.16 & 0.05 & 21.53 & 0.06 \\
\hline 14:03:54.772 & $+54: 22: 01.28$ & 25.45 & 0.05 & 23.76 & 0.04 & 21.54 & 0.05 \\
\hline 14:03:53.027 & $+54: 21: 34.49$ & 25.34 & 0.05 & 23.56 & 0.03 & 21.55 & 0.04 \\
\hline 14:03:53.238 & $+54: 21: 51.47$ & 25.13 & 0.07 & 23.50 & 0.03 & 21.59 & 0.04 \\
\hline 14:03:51.968 & $+54: 21: 33.02$ & 25.84 & 0.07 & 24.06 & 0.03 & 21.60 & 0.04 \\
\hline 14:03:52.200 & $+54: 21: 36.24$ & 25.33 & 0.07 & 23.58 & 0.07 & 21.60 & 0.09 \\
\hline 14:03:35.520 & $+54: 22: 17.92$ & 25.22 & 0.07 & 23.61 & 0.02 & 21.62 & 0.04 \\
\hline 14:03:53.763 & $+54: 21: 53.60$ & 25.77 & 0.07 & 24.15 & 0.04 & 21.62 & 0.06 \\
\hline
\end{tabular}


Table 2: (continued)

\begin{tabular}{|c|c|c|c|c|c|c|c|}
\hline $\mathrm{X}$ & $\bar{Y}$ & F435W & err & F555W & err & F814W & err \\
\hline 14:03:54.566 & $+54: 21: 45.77$ & 25.26 & 0.04 & 23.57 & 0.02 & 21.63 & 0.03 \\
\hline 14:03:56.658 & $+54: 22: 22.04$ & 25.63 & 0.05 & 23.82 & 0.03 & 21.64 & 0.04 \\
\hline 14:03:52.822 & $+54: 21: 44.51$ & 25.45 & 0.04 & 23.84 & 0.03 & 21.64 & 0.04 \\
\hline 14:03:54.379 & $+54: 21: 48.41$ & 25.84 & 0.06 & 24.04 & 0.02 & 21.65 & 0.03 \\
\hline 14:03:49.694 & $+54: 21: 13.17$ & 25.89 & 0.07 & 24.15 & 0.04 & 21.65 & 0.05 \\
\hline 14:03:54.057 & $+54: 22: 02.87$ & 25.09 & 0.05 & 23.48 & 0.03 & 21.66 & 0.04 \\
\hline 14:03:54.617 & $+54: 22: 56.56$ & 25.32 & 0.04 & 23.69 & 0.02 & 21.66 & 0.03 \\
\hline 14:03:37.847 & $+54: 22: 02.26$ & 25.47 & 0.05 & 23.81 & 0.03 & 21.66 & 0.04 \\
\hline 14:03:43.282 & $+54: 22: 26.49$ & 25.72 & 0.05 & 24.01 & 0.02 & 21.67 & 0.03 \\
\hline 14:03:51.967 & $+54: 22: 53.10$ & 25.58 & 0.07 & 23.98 & 0.05 & 21.67 & 0.06 \\
\hline 14:03:52.185 & $+54: 21: 18.89$ & 25.65 & 0.07 & 24.04 & 0.05 & 21.67 & 0.06 \\
\hline 14:03:53.076 & $+54: 21: 35.38$ & 25.73 & 0.06 & 23.86 & 0.04 & 21.68 & 0.04 \\
\hline 14:03:53.446 & $+54: 22: 02.87$ & 25.87 & 0.16 & 23.79 & 0.04 & 21.68 & 0.05 \\
\hline 14:03:52.282 & $+54: 21: 58.84$ & 26.00 & 0.08 & 24.05 & 0.04 & 21.68 & 0.05 \\
\hline 14:03:53.326 & $+54: 21: 52.79$ & 25.32 & 0.08 & 23.65 & 0.06 & 21.69 & 0.08 \\
\hline 14:03:51.768 & $+54: 21: 23.80$ & 25.75 & 0.07 & 23.90 & 0.04 & 21.70 & 0.05 \\
\hline 14:03:51.756 & $+54: 21: 23.63$ & 25.76 & 0.10 & 24.01 & 0.06 & 21.70 & 0.06 \\
\hline 14:03:56.264 & $+54: 21: 53.28$ & 25.64 & 0.11 & 24.04 & 0.04 & 21.70 & 0.05 \\
\hline 14:03:50.450 & $+54: 21: 54.07$ & 25.53 & 0.06 & 23.75 & 0.03 & 21.70 & 0.05 \\
\hline 14:03:54.868 & $+54: 21: 59.07$ & 25.89 & 0.09 & 24.07 & 0.03 & 21.72 & 0.04 \\
\hline 14:03:53.418 & $+54: 21: 51.24$ & 25.52 & 0.06 & 23.77 & 0.03 & 21.73 & 0.04 \\
\hline 14:03:55.619 & $+54: 21: 56.82$ & 25.72 & 0.06 & 23.94 & 0.03 & 21.75 & 0.04 \\
\hline 14:03:51.115 & $+54: 21: 27.39$ & 25.83 & 0.06 & 24.12 & 0.03 & 21.76 & 0.04 \\
\hline 14:03:53.779 & $+54: 21: 59.47$ & 25.50 & 0.05 & 23.63 & 0.04 & 21.76 & 0.05 \\
\hline 14:03:39.781 & $+54: 21: 07.18$ & 25.76 & 0.07 & 23.91 & 0.03 & 21.76 & 0.05 \\
\hline 14:03:54.274 & $+54: 21: 40.79$ & 25.83 & 0.05 & 24.21 & 0.04 & 21.80 & 0.05 \\
\hline 14:03:51.490 & $+54: 21: 21.41$ & 25.64 & 0.05 & 24.02 & 0.03 & 21.82 & 0.04 \\
\hline 14:03:43.061 & $+54: 22: 28.83$ & 25.60 & 0.06 & 23.94 & 0.04 & 21.82 & 0.05 \\
\hline 14:03:52.248 & $+54: 21: 32.04$ & 25.50 & 0.05 & 23.87 & 0.02 & 21.83 & 0.03 \\
\hline 14:03:37.785 & $+54: 22: 13.83$ & 25.93 & 0.04 & 24.15 & 0.03 & 21.83 & 0.04 \\
\hline 14:03:40.408 & $+54: 23: 25.01$ & 25.58 & 0.06 & 23.91 & 0.03 & 21.83 & 0.05 \\
\hline 14:03:52.086 & $+54: 21: 37.68$ & 25.66 & 0.06 & 23.92 & 0.02 & 21.84 & 0.03 \\
\hline 14:03:52.948 & $+54: 21: 42.57$ & 25.51 & 0.06 & 23.88 & 0.03 & 21.84 & 0.04 \\
\hline 14:03:51.309 & $+54: 21: 20.38$ & 25.57 & 0.07 & 23.95 & 0.06 & 21.84 & 0.07 \\
\hline 14:03:42.129 & $+54: 23: 49.29$ & 25.39 & 0.08 & 23.75 & 0.05 & 21.86 & 0.06 \\
\hline 14:03:53.636 & $+54: 21: 38.60$ & 25.82 & 0.04 & 24.17 & 0.02 & 21.87 & 0.03 \\
\hline 14:03:53.212 & $+54: 21: 59.41$ & 25.44 & 0.06 & 23.82 & 0.04 & 21.87 & 0.05 \\
\hline 14:03:54.313 & $+54: 22: 04.51$ & 25.85 & 0.15 & 24.05 & 0.04 & 21.88 & 0.05 \\
\hline 14:03:53.181 & $+54: 21: 45.97$ & 25.76 & 0.05 & 24.08 & 0.04 & 21.89 & 0.05 \\
\hline
\end{tabular}


clustering of WR candidates in the central core of the largest star-forming complex in the field. Thousands of WR and RSG candidates, and hundreds of spectrographically confirmed WR stars will be reported in future papers in this series.

This research is based on NASA/ESA Hubble Space Telescope observations obtained at

the Space Telescope Science Institute, which is operated by the Association of Universities for Research in Astronomy Inc. under NASA contract NAS5-26555. JLB and MMS acknowledge the interest and generous support of Hilary and Ethel Lipsitz. AFJM and LD are grateful to NSERC (Canada) and FQRNT (Quebec) for financial assistance. We thank Or Graur for suggestions on displaying the contours shown in Figure 10.

\section{REFERENCES}

Adamo, A., Östlin, G., Zackrisson, E., et al. 2011, MNRAS, 415, 2388

Armandroff, T. E., \& Massey, P. 1985, ApJ, 291, 685

Barmby, P., Kuntz, K. D., Huchra, J. P., \& Brodie, J. P. 2006, AJ, 132, 883

Bastian, N., Adamo, A., Gieles, M., et al. 2012, MNRAS, 419, 2606

Bersten, M.C., Benvenuto, O.G., Nomoto, K., Ergon, M., Folatelli, G., Sollerman, J., Benetti, S., Botticella, M.T., Fraser, M., Kotak, R., Maeda, K., Ochner, P., \& Tomasella, L. 2012, ApJ, 757, 31

Bianchi, L., Thilker, D. A., Burgarella, D., et al. 2005, ApJ, 619, L71

Bibby, J.L., \& Crowther, P.A. 2010, MNRAS, 405, 2737

Bibby, J.L., \& Crowther, P.A. 2012, MNRAS, 420, 3483

Cardelli, J.A., Clayton, G.C. \& Mathis, J.S., ApJ, 345, 245

Chen, C.H.R., Chu, Y.H., \& Johnson, K.E., AJ, 619, 779

Clark, J. S., Negueruela, I., Crowther, P. A., \& Goodwin, S. P. 2005, A\&A, 434, 949

Clark, J.S., Castro, N., Garcia, M., Herrero, A., Najarro, F., Negueruela, I., Ritchie, B.W., \& Smith, K.T. 2012, å, 541, 146

Crockett, R.M., Smartt, S.J., Eldridge, J.J., Mattila, S., Young, D.R., Pastorello, A., Maund, J.R., Benn, C.R., \& Skillen, I. 2007, MNRAS, 381, 835 
Crowther, P. A., Beck, S. C., Willis, A. J., et al. 1999, MNRAS, 304, 654

Crowther, P. A., Drissen, L., Abbott, J. B., Royer, P., \& Smartt, S. J. 2003, A\&A, 404, 483

Crowther, P. A., Hadfield, L. J., Schild, H., \& Schmutz, W. 2004, A\&A, 419, L17

Crowther, P. A. 2007, ARA\&A, 45, 177

Crowther, P. A., Barnard, R., Carpano, S., et al. 2010, MNRAS, 403, L41

Crowther, P. A. 2013, MNRAS, 428, 1927

de Koter, A., Heap, S.R., \& Hubeny, I. 1998, ApJ, 509, 879

Drissen, L., Shara, M. M., \& Moffat, A. F. J. 1991, AJ, 101, 1659

Drissen, L., Moffat, A. F. J., \& Shara, M. M. 1993, AJ, 105, 1400

Drissen, L., Moffat, A. F. J., Walborn, N. R., \& Shara, M. M. 1995, AJ, 110, 2235

Drissen, L., Roy, J.-R., Moffat, A. F. J., \& Shara M.M. 1999, AJ, 117, 1249

Drissen, L., Roy, J.-R., Robert, C., Devost, D., \& Doyon, R. 2000, AJ, 119, 688

Eldridge, J. J., Fraser, M., Smartt, S.J., Maund, J.R., \& Crockett, M.R., Submitted to MNRAS, arXiv:1301.1975

Freedman, W. L., \& Madore, B. F. 1988, ApJ, 332, L63

Fruchter, A. S., Levan, A. J., Strolger, L., et al. 2006, Nature, 441, 463

Gazak, J. Z., Bastian, N., Kudritzki, R.-P., et al. 2012, MNRAS, L41

Georgy, C., Ekström, S., Meynet, G., et al. 2012, A\&A, 542, A29

Hadfield, L. J., Crowther, P. A., Schild, H., \& Schmutz, W. 2005, A\&A, 439, 265

Hadfield, L. J., \& Crowther, P. A. 2007, MNRAS, 381, 418

Hamann, W.-R., Koesterke, L., \& Wessolowski, U. 1995, A\&AS, 113, 459

Henry, R. B. C., \& Howard, J. W. 1995, ApJ, 438, 170

Ho, L. C., \& Filippenko, A. V. 1996, ApJ, 466, L83

Hodge, P.W., Gurwell, M., Goldader, J.D., \& Kennicutt, R.C.Jr, ApJS, 73, 661 
Hunter, D. A., Shaya, E. J., Holtzman, J. A., et al. 1995, ApJ, 448, 179

Hunter, D. A., Baum, W. A., O’Neil, E. J., Jr., \& Lynds, R. 1996, ApJ, 456, 174

Kelly, P. L., Kirshner, R. P., \& Pahre, M. 2008, ApJ, 687, 1201

Kennicutt, R. C., Jr., Bresolin, F., \& Garnett, D. R. 2003, ApJ, 591, 801

Larsen, S. S., de Mink, S. E., Eldridge, J. J., et al. 2011, A\&A, 532, A147

Lee, J.C., et al., 2009, ApJ, 706, 599

Leitherer, C. 1997, Revista Mexicana de Astronomia y Astrofisica Conference Series, 6, 114

Leitherer, C., \& Heckman, T. M. 1995, ApJS, 96, 9

Leloudas, G., Sollerman, J., Levan, A. J., et al. 2010, A\&A, 518, A29

Levesque, E. M. 2010, New A Rev., 54, 1

Maeder, A., \& Meynet, G. 1994, A\&A, 287, 803

Malumuth, E. M., Waller, W. H., \& Parker, J. W. 1996, AJ, 111, 1128

Maoz, D., Ho, L. C., \& Sternberg, A. 2001, ApJ, 554, L139

Martin, P., \& Belley, J. 1996, ApJ, 468, 598

Massey, P. 1998, ApJ, 501, 153

Massey, P., \& Hunter, D. A. 1998, ApJ, 493, 180

Massey, P., \& Johnson, O. 1998, ApJ, 505, 793

Massey, P., Silva, D.R., Levesque, E.M., Plez, B., Olsen, K.A., Clayton, G.C., Meynet, G., \& Maeder, A. 2009, ApJ, 703, 420

Mattila, S., Smartt, S.J., Maund, J., Benetti, S., \& Ergon, M. 2010, arXiv, 1011.5494

Maund, J.R., Fraser, M., Ergon, M., Pastorello, A., Smartt, S.J., Sollerman, J, Benetti, S., Botticella, M.T., Bufano, F., Danzinger, I.J., Kotak, R., Magill, L., Stephens, A.W., Valenti, S. 2011, ApJ, 739, 37

Meurer, G. R. 1995, Nature, 375, 742 
Meynet, G., Georgy, C., Hirschi, R., et al. 2011, Bulletin de la Societe Royale des Sciences de Liege, 80, 266

Moffat, A..J., \& Shara, M.M. 1983, ApJ, 273, 544

Moffat, A. F. J., Drissen, L., \& Shara, M. M. 1994, ApJ, 436, 183

Neugent, K. F., Massey, P., \& Georgy, C. 2012, ApJ, 759, 11

Neugent, K. F., \& Massey, P. 2011, ApJ, 733, 123

Podsiadlowski, Ph., Joss, P.C., \& Hsu, J.J.L. 1992, ApJ, 391, 246

Schlafly, E.F., \& Finkbeinger, D.P. 2011, ApJ, 737, 103

Scowcroft, V., Bersier, D., Mould, J. R., \& Wood, P. R. 2009, MNRAS, 396, 1287

Shappee, B. J. \& Stanek, K. Z. 2011, ApJ, 733, 124

Shara, M. M., Moffat, A. F. J., Smith, L. F., et al. 1999, AJ, 118, 390

Sidoli, F., Smith, L. J., \& Crowther, P. A. 2006, MNRAS, 370, 799

Sivan, J.-P., Maucherat, A. J., Petit, H., \& Comte, G. 1990, A\&A, 237, 23

Smartt, S. J., Maund, J. R., Hendry, M. A., et al. 2004, Science, 303, 499

Smartt, S.J. 2009, ARA\&A, 47, 63

Stetson, P. 1987, PASP, 99, 191

Vacca, W. D., Sheehy, C. D., \& Graham, J. R. 2007, ApJ, 662, 272

van der Hucht, K. A., Hidayat, B., Admiranto, A. G., Supelli, K. R., \& Doom, C. 1988, A\&A, 199, 217

Walborn, N. R., \& Blades, J. C. 1997, ApJS, 112, 457

Whitmore, B. C., \& Schweizer, F. 1995, AJ, 109, 960

Whitmore, B. C., Gilmore, D., Leitherer, C., et al. 2005, AJ, 130, 2104

Whitmore, B. C., Chandar, R., Kim, H., et al. 2011, ApJ, 729, 78

Yoon, S.C., Woosley, S.E., \& Langer, N. 2010, ApJ, 725, 940

Yoon, S.C., Gräfener, G., Vink, J.S., Kozyreva, A., \& Izzard, R.G. 2012, A\&A, 554, 11 
This preprint was prepared with the AAS LATEX macros v5.2. 